\title{
Noradrenergic Responsiveness Supports Selective Attention across the Adult Lifespan
}

\author{
${ }^{\circledR}$ Martin J. Dahl, ${ }^{1}{ }^{\circledR}$ Mara Mather, ${ }^{2}{ }^{\circledR}$ Myriam C. Sander, ${ }^{1}$ and ${ }^{\circledR}$ Markus Werkle-Bergner ${ }^{1}$ \\ ${ }^{1}$ Center for Lifespan Psychology, Max Planck Institute for Human Development, Berlin 14195, Germany, and ${ }^{2}$ Davis School of Gerontology, \\ University of Southern California, Los Angeles, California 90089
}

Selectively attending to relevant information while blocking out distractors is crucial for goal-directed behavior, yet with advancing age, deficits emerge in attentional selectivity. Decrements in attention have been associated with altered noradrenergic activity in animals. However, research linking noradrenergic functioning to attention in aging humans is scarce, likely reflecting long-standing methodological challenges in noninvasive assessments. We studied whether age-related differences in the noradrenergic system predict differences in attention. We measured pupil dilation, a noninvasive marker of arousalrelated norepinephrine (NE) release, while concurrently recording the EEG of male younger $(N=39 ; 25.2 \pm 3.2$ years) and older adults $(N=38 ; 70.6 \pm 2.7$ years $)$. Arousal was modulated on a trial-by-trial basis using fear-conditioned $\left(\mathrm{CS}^{+}\right)$stimuli. During conditioning, pupil and EEG markers related to heightened arousal were identified. Afterward, in a dichotic listening task, participants were cued to direct attention to either the left or right ear while highly similar syllable pairs were presented simultaneously to both ears. During the dichotic listening task, presentation of fear-conditioned stimuli reinstated the acquired arousal response, as reflected in pupil and EEG $\alpha-\beta$ band responses. Critically, pupil dilation to $\mathrm{CS}^{+}$was correlated with stronger EEG $\boldsymbol{\alpha}-\boldsymbol{\beta}$ desynchronization, suggesting a common dependence on NE release. On a behavioral level, stronger arousal reactions were associated with better attention. In particular, structural equation modeling revealed that the responsiveness of the NE system is associated with attention on a latent construct level, measured by several indicator tasks. Overall, our results suggest that the responsiveness of the NE system supports attention across the lifespan.

Key words: cognitive aging; locus coeruleus; noradrenaline; norepinephrine; rhythmic neural activity; selective attention

Significance Statement

In old age, the ability to selectively process relevant aspects of the environment fades. Animal research suggests that the neuromodulator norepinephrine helps to maintain selective attention. We tested younger and older adults across a variety of attention tasks. In addition, we used arousing stimuli to experimentally activate participants' noradrenergic system while recording pupillometry and EEG to infer its functional capacity. Older adults showed compromised attention and reduced noradrenergic responsiveness as indicated by interrelated pupil and EEG markers. Crucially, in both age groups, a more responsive noradrenergic system was strongly associated with attention. Our findings link animal and human studies on the neural underpinning of attention in aging and underscore the importance of the noradrenergic system in late-life cognition.

Received Feb. 19, 2019; revised Mar. 5, 2020; accepted Mar. 26, 2020.

Author contributions: M.J.D., M.C.S., M.M., and M.W.-B. designed research; M.J.D. performed research; M.J.D. and M.W.-B. analyzed data; M.J.D. and M.W.-B. wrote the first draft of the paper; M.J.D., M.M., M.C.S., and M.W.-B. edited the paper; M.J.D., M.M., M.C.S., and M.W.-B. wrote the paper.

The authors declare no competing financial interests.

M.W.-B. was supported by German Research Foundation (Deutsche Forschungsgemeinschaft) WE 4269/5-1, and Jacobs Foundation Early Career Research Fellowship 2017-2019. M.C.S. was supported by Max Planck Society and the German Research Foundation (Deutsche Forschungsgemeinschaft) MINERVA Program BR 4918/2-1. M.J.D. is a fellow of the International Max Planck Research School on the Life Course and was supported by a Sonnenfeld-Foundation stipend. M.M. was supported by an Alexander von Humboldt fellowship, a Max Planck Sabbatical Award, and National Institutes of Health Grant R01AG025340. We thank all participants and student assistants involved in the study, and in particular, I. Boux, Y.-J. Yi, M. Lawes, and N. Doehring, for valuable help during data collection and preprocessing.

Correspondence should be addressed to Martin J. Dahl at dahl@mpib-berlin.mpg.de or Markus WerkleBergner at werkle@mpib-berlin.mpg.de.

https://doi.org/10.1523/JNEUROSCI.0398-19.2020

Copyright $\odot 2020$ the authors

\section{Introduction}

Daily situations confront us with a plethora of competing sensory inputs that far exceed neural processing capacities; thus, prioritization and selection are essential for adaptive behavior (Desimone and Duncan, 1995). Impaired attentional selection in aging (Kennedy and Mather, 2019) has been linked to deficient neuromodulation (Li et al., 2001; Bäckman et al., 2006). The neuromodulator norepinephrine (NE) is strongly implicated in attentional processes that facilitate the processing of relevant information (Berridge and Waterhouse, 2003). First, increased NE release is associated with the transition to, and the maintenance of, an activated cortical and behavioral state, as evident in a desynchronized (high-frequency, low-amplitude) EEG and alert waking (sometimes termed arousal) (Carter et al., 2010; 
McGinley et al., 2015; Neves et al., 2018). In the waking state, fast, burst-like (phasic) and slow, rhythmic (tonic) firing patterns of the locus coeruleus (LC), the primary cortical NE source, have been tied to focused attention and distractibility, respectively (Aston-Jones and Cohen, 2005). Further, a series of pharmacological and lesion studies demonstrated that, via actions at $\alpha_{2 \mathrm{~A}^{-}}$ adrenoceptors in the PFC, NE facilitates top-down selective attention (Robbins and Arnsten, 2009). In the sensory cortices, phasic NE release interacts with local glutamate levels to allow the selective processing of currently relevant representations, mediated via $\alpha_{2 A^{-}}$and $\beta$-adrenoceptors (Mather et al., 2016; Gelbard-Sagiv et al., 2018). Finally, NE has been linked to the reorienting and switching of attention via disruption of the dorsal and activation of the ventral attention network (Bouret and Sara, 2005; Corbetta et al., 2008). In line with these links between $\mathrm{NE}$ and attention, recent theories of both healthy (Mather and Harley, 2016) and pathologic (Weinshenker, 2018; Satoh and Iijima, 2019) cognitive aging have proposed a prominent role of the LC-NE system in late-life cognition. However, LC's anatomic location in the brainstem, adjacent to the ventricular system, and its widespread, unmyelinated axons expose it to blood- and CSFbound toxins, making it vulnerable to neurodegeneration (Mather and Harley, 2016; Liu et al., 2019), with potentially wide-ranging consequences. For instance, Wilson et al. (2013) reported an association between LC's structural integrity, as assessed postmortem via autopsy, and longitudinal cognitive decline in aging (compare Hämmerer et al., 2018; Betts et al., 2019; Dahl et al., 2019b). However, the question of how LC's functional characteristics (i.e., its capacity to respond to behaviorally relevant information) are linked to attention in aging humans is still unresolved. Long-standing technical challenges in the noninvasive assessment of LC-NE activity in vivo (compare Astafiev et al., 2010) have contributed to this lack of information. However, recently, multiple independent studies (Joshi et al., 2016; Reimer et al., 2016; Breton-Provencher and Sur, 2019; Zerbi et al., 2019) demonstrated that pupil dilation in the absence of interfering visual input serves as valid, noninvasive proxy for LC activity. In addition, use of optogenetics established a causal link between phasic LC activity and event-related EEG responses (i.e., P300 event-related potential [ERP]) (Vazey et al., 2018). Moreover, EEG reveals fluctuations in cortical states (i.e., global EEG de/activation as reflected in a de/synchronized EEG) that have been associated with LC activity (McCormick et al., 1991).

In this study, we thus used a multimodal assessment to evaluate individual differences in selective attention among younger adults (YA) and older adults (OA) and their dependence on functional characteristics of the LC-NE system. In order to experimentally induce LC activity, we made use of LC-NE's wellestablished role in fear processing (Szabadi, 2012; Uematsu et al., 2017; Lee et al., 2018; Likhtik and Johansen, 2019). We hypothesized that the functional capacity of the LC-NE system, as assessed by simultaneous, interrelated pupil and EEG responses, would be closely associated with individual differences in selective attention. In sum, the overall goal of this study was to extend our knowledge about the role of the LC-NE system in human cognitive aging by generating a multimodal, noninvasive index of LC functioning and linking it to attention abilities in YA and $\mathrm{OA}$.

\section{Materials and Methods \\ Study design}

Data were collected within the framework of a larger study investigating the interplay of neurophysiological indices of LC-NE activity and their association with selective attention in YA and OA. Only aspects of the study that are relevant to the current analyses are introduced in detail below.

Participants were invited on 3 successive days (days 1-3) for individual assessments that spanned $\sim 4 \mathrm{~h}$ on day 1 and day 2 and $2 \mathrm{~h}$ on day 3 . Time of assessment (morning, afternoon, evening) was kept constant across sessions within participants.

In short, on the first day, participants completed a neuropsychological selective attention battery as well as an assessment of fear conditionability (Fig. 1a,c) while pupil dilation was recorded. To adapt auditory stimuli during later attention assessments for hearing thresholds, we assessed hearing acuity (on day 1 for YA and on a separate occasion preceding day 1 for $\mathrm{OA}$ ). On the second day, we concurrently recorded pupil dilation and EEG while participants underwent another fear conditioning session and an in-depth evaluation of their auditory-selective attention performance (Fig. 1b). The last day comprised a final fear conditioning session while recording pupil dilation (Fig. 1c) and an MRI assessment (compare Dahl et al., 2019b). The study was approved by the ethics committee of the German Psychological Association and was conducted in accordance with relevant guidelines and regulations.

\section{Participants}

Forty-one YA participated in the study. Two of these (4.88\%; aged: 24.59 and 29.02 years) were not reinvited after day 1 due to low-quality eyetracking data, reducing the final sample to 39 YA (mean \pm SD age: $25.23 \pm 3.23$ years; range: $20.17-31$ years; $100 \%$ male). In addition, 38 OA took part in the experiments (age: $70.61 \pm 2.71$ years; range: 65.50 75.92 years; $100 \%$ male). All participants were healthy, MRI-compatible, right-handed, fluent German speakers with normal or corrected-to-normal vision who provided written informed consent and were reimbursed for participation. Intake of centrally active drugs, and in particular medication directly influencing the LC-NE system (e.g., $\beta$-blockers), precluded participation. The current study only tested male subjects due to our pilot data demonstrating reliable sex differences in the stimulation intensity participants selected for the fear conditioning sessions. Previous research indicates sex differences in the capability to learn and maintain fear responses during conditioning (Merz et al., 2018; but for evidence for sex-specific expressions of fear learning, see also Gruene et al., 2015; Voulo and Parsons, 2017). Some of these differences in fear learning may be associated with sex differences in the LC-NE system (Bangasser et al., 2016; Mulvey et al., 2018). To limit the number of control variables, we decided to test male participants; however, we would like to underscore the necessity to follow-up on the reported findings, including both sexes. Descriptive characteristics of the two age groups are provided in Table 1. Both groups showed comparable educational levels and did not differ reliably on a brief dementia screening (Mini Mental State Examination; Folstein et al., 1975). All participants scored above the commonly used Mini Mental State Examination cutoff of 26 points. In line with previous reports (e.g., Passow et al., 2012, 2014), OA demonstrated higher scores on a test of verbal knowledge (Spot a Word; Lehrl, 1977) and increased age-related hearing loss. On average, OA selected higher intensities as unpleasant unconditioned stimulus (US) for the fear conditioning procedure, presumably reflecting age-related differences in skin conductivity (Chamberlin et al., 2011).

\section{Experimental procedures and stimuli}

Neuropsychological attention assessment. On the first day of the experiment, participants completed a multimodal, standardized neuropsychological attention assessment comprising the D2 test of attention (Brickenkamp and Zillmer, 1998), Digit-Symbol-Substitution Test (DSST) (Wechsler, 1981), and auditory Digit Sorting Task (DST) (compare Kray and Lindenberger, 2000).

The D2 test is a paper-and-pencil cancellation task asking participants to cross out any letter $d$ with two marks (") around, above, or below it from a stream of highly similar surrounding distractors (e.g., $p$ with two marks or $d$ with only one mark). Participants were granted $20 \mathrm{~s}$ to complete each of a total of 12 lists of items. The difference between processed items and committed errors (errors of omission and errors of commission) across lists was taken as a measure of attention. 


\section{a Conditioning}

\section{b Dichotic listening}
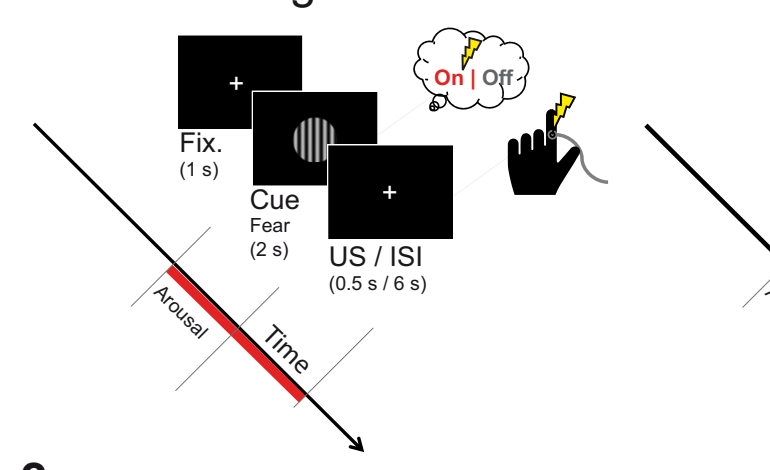

C

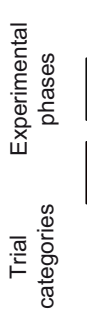

Day 10
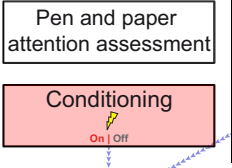

Conditioning

(Day 1; Day 2; Day 3)
Day 2
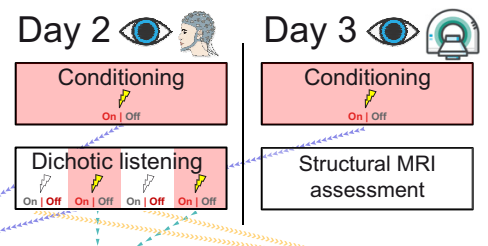

assessment

Figure 1. Experimental procedure of the $(\boldsymbol{a})$ fear conditioning session, $(\boldsymbol{b})$ dichotic listening task, and $(\boldsymbol{c})$ resulting trial categories. $\boldsymbol{a}$, Each conditioning trial began with a $1 \mathrm{~s}$ baseline interval during which a white fixation cross was presented. Then, either the conditioned (CS ${ }^{+}$) or perceptually matched control stimulus (CS ; horizontal or vertical Gabor patch) was displayed for $2 \mathrm{~s}$. In $\mathrm{CS}^{+}$trials, on offset of the Gabor patch, a mild electric shock was applied to the participant's finger. After $6 \mathrm{~s}$ of fix cross presentation (ITI), the next trial started. $\boldsymbol{b}$, Importantly, the beginning of trials in the dichotic listening task was identical to the fear conditioning session. Upon offset of the CS, an attention cue ( 2 s) indicated which ear participants should focus on. Tightly synchronized syllable pairs were then presented simultaneously to the left and right ear $(0.5 \mathrm{~s}$ ), and participants indicated by button press which syllable they heard on the cued ear (up to 3 s). c, Participants underwent fear conditioning sessions (compare $\boldsymbol{a}$ ) on each day of the experiment while pupil dilation was recorded (Conditioning; blue). The arousal manipulated dichotic listening task (compare $\boldsymbol{b}$ ) was completed once on the second day while both pupil dilation and EEG were recorded. Interleaved after each break of the dichotic listening task, participants were reconditioned (compare $\boldsymbol{a}$ ) to prevent extinction of the fear response (Reconditioning; teal). During re/conditioning, participants received electrical stimulation (US); thus, the observed responses may represent a mixture of fear and somatosensory responses. In contrast, during the dichotic listening task, no shocks were applied; thus, observed responses to the arousal manipulation (CS ${ }^{+}$vs $\left(S^{-}\right)$indicate the reinstatement of the fear response (Reinstatement; orange). On the first day of the experiment, participants additionally completed a pen-and-paper attention assessment; whereas on the last day, structural MRI data were recorded. Fix., Fixation; ISI, interstimulus interval. Numbers in parentheses indicate presentation times in seconds.

Table 1. Descriptive statistics for $\mathrm{YA}$ and $\mathrm{OA}^{a}$

\begin{tabular}{lrlrrr}
\hline Measure & YA $(n=39)$ & OA $(n=38)$ & \multicolumn{1}{c}{$U$} & \multicolumn{1}{c}{$Z$} \\
\hline Age (yr) & $25.231 \pm 0.516$ & $70.614 \pm 0.440$ & 780 & -7.546 & $<0.001$ \\
Education (yr) & $16.171 \pm 0.406$ & $17.263 \pm 0.560$ & 1320 & -1.487 & 0.137 \\
Hearing level (dB) & $8.027 \pm 0.758$ & $19.626 \pm 1.075$ & 907 & -6.251 & $<0.001$ \\
Shock intensity (mA) & $0.3 \pm 0.026$ & $0.4 \pm 0.024$ & 1211 & -3.227 & 0.001 \\
Spot a Word (correct rows) & $21.487 \pm 0.551$ & $29.158 \pm 0.382$ & 834 & -7.021 & $<0.001$ \\
Mini Mental State & $29.410 \pm 0.120$ & $29.053 \pm 0.181$ & 1631 & 1.216 & 0.224 \\
\multicolumn{1}{c}{ Examination (points) } & & & & &
\end{tabular}

${ }^{a}$ For $Y A$ and $O A$, group means \pm 1 SEM are reported. Age group comparisons are based on nonparametric Mann-Whitney $U$ tests. Hearing level is averaged across frequencies $(250-3000 \mathrm{~Hz})$ and ears. Shock intensity denotes the intensity participants individually defined as uncomfortable yet not painful on day 2 .

During the DSST, participants were presented with a list of digitsymbol pairs (e.g., 1: -; 2: $\perp ;[\ldots]$ 9: =) along with a list of digits. Participants were asked to write down the corresponding symbol under each digit as quickly and accurately as possible. The number of correctly copied symbols within $90 \mathrm{~s}$ was taken as an index of attention.

In the auditory DST, we auditorily presented participants with a stream of numbers (e.g., 2-7-5) ranging from 3 to 8 digits. Participants then had to write down the numbers sorted according to numerosity (e.g., 2-5-7). The sum of correctly reported answers across all trials was taken as a measure of attention.

Fear conditioning. To experimentally activate the LC-NE system (Rasmussen and Jacobs, 1986; Szabadi, 2012; Uematsu et al., 2017; Likhtik and Johansen, 2019) on each assessment day (days 1-3), participants completed a brief fear conditioning session in which they learned the association between a visual stimulus and an aversive electrical shock (compare Lee et al., 2018; Fig. 1a). During this phase, either a horizontal or vertical sinusoidal luminance pattern (i.e., Gabor patch; $\mathrm{CS}^{+}$) was paired with a US (shock). The other pattern was never paired with the US and served as perceptually matched control stimulus $\left(\mathrm{CS}^{-}\right)$; the association between pattern orientation (horizontal/vertical) and shock was kept constant within subject across days and was counterbalanced within age groups (YA: 21:18; OA: 20:18). This design guaranteed a matched luminance of $\mathrm{CS}^{+}$and $\mathrm{CS}^{-}$, whereas the former acquired the behavioral relevance to stimulate LC-NE activity (Szabadi, 2012). Each conditioning session comprised 40 trials, which started with a central white fixation cross on a black background (baseline; $1 \mathrm{~s}$ ), followed by the visual stimulus $\left(2 \mathrm{~s} ; \mathrm{CS}^{+}\right.$or $\mathrm{CS}^{-}$; Fig. 1a). After offset of the visual stimulus, the shock was applied in $\mathrm{CS}^{+}$trials for $0.5 \mathrm{~s}$ with a $80 \%$ reinforcement schedule (i.e., $0 \mathrm{~s}$ trace conditioning) using a ring electrode connected to a bipolar current stimulator (DS5; Digimeter) that was taped either to participants' left or right index finger (hand assignment was constant within subject across days and counterbalanced within age groups: YA: 19:20; OA: 19:19). The intertrial interval (ITI; white fixation cross) was set to $6 \mathrm{~s}$ to allow sufficient time for the pupil to return to baseline in $\mathrm{CS}^{+}$trials (compare Lee et al., 2018). The conditioning phase consisted of $20 \mathrm{CS}^{+}$and $20 \mathrm{CS}^{-}$trials that were presented in pseudorandomized order. After half of the trials, participants had a self-paced break during which they were asked to indicate which of the two visual stimuli (horizontal; vertical) was paired with the shock and how many shocks were delivered (i.e., manipulation check). On each day, before the experiment, participants individually selected an intensity for the US, which they perceived as unpleasant but not painful (compare Lee et al., 2018; Table 1). During all conditioning sessions, gaze position and pupil dilation were recorded, interfering sensory input was minimized and central fixation was enforced (see below).

During data collection, for one OA, assignment of Gabor patch orientation (horizontal/vertical) to $\mathrm{CS}$ condition $\left(\mathrm{CS}^{-} / \mathrm{CS}^{+}\right)$was switched 
between the first and second day of assessment. Since this error worked against finding a reliable difference in responses to $\mathrm{CS}^{-}$and $\mathrm{CS}^{+}$, we decided not to exclude this subject.

Dichotic listening task. On the second assessment day, we probed selective auditory attention by cueing participants to focus attention to either the left (focus left condition [FL]) or right (focus right condition [FR]) ear while highly similar consonant-vowel syllable pairs were presented dichotically (i.e., simultaneously one stimulus to the left and one to the right ear). Only syllables played to the cued ear should be reported, whereas distractor stimuli should be ignored. To indicate their response, after a brief delay, participants selected the target syllable from three visually displayed response options (including the target, distractor, and one highly similar novel, i.e., not presented, syllable; Fig. 1b).

Within attentional conditions (FL and FR), we manipulated participants' arousal level on a trial-by-trial basis. In particular, each trial started with a central white fixation cross on a black background (baseline; $1 \mathrm{~s}$ ), followed by one of the conditioned stimuli $\left(2 \mathrm{~s} ; \mathrm{CS}^{+}\right.$or $\left.\mathrm{CS}^{-}\right)$. After offset of the CS, a letter was centrally displayed cuing participants to adapt their attentional focus ( $2 \mathrm{~s} ; L$ for FL; $R$ for FR). Next, a syllable pair was presented dichotically for $0.5 \mathrm{~s}$. After a delay of $0.5 \mathrm{~s}$, a recognition matrix (containing the target, distractor, and one novel syllable) was visually displayed for up to $3 \mathrm{~s}$ and participants indicated by button press which syllable they heard in the cued ear. Response hand assignment was counterbalanced within age groups (YA: 20:19; OA: 19:19; shocks were never applied to the response hand). The ITI was set to $0.5 \mathrm{~s}$ and consisted of a white fixation cross. Matched trial timing between fear conditioning and dichotic listening tasks ( $1 \mathrm{~s}$ baseline; $2 \mathrm{~s} \mathrm{CS} ; \sim 6 \mathrm{~s}$ until next trial) allows a comparison of arousal responses across both tasks (Fig. 1).

Twelve consonant-vowel syllable pairs consisting of syllables of voiced $(/ \mathrm{b} /, / \mathrm{d} /, / \mathrm{g} /)$ or unvoiced $(/ \mathrm{p} /, / \mathrm{t} /, / \mathrm{k} /)$ consonants combined with the vowel $/ \mathrm{a} /$ served as auditory stimuli in the dichotic listening task. Each pair contained two syllables with the same voicing that were matched for onset times (compare Hugdahl et al., 2009; Westerhausen et al., 2009). To account for age-related hearing loss, syllable pairs were presented at an individually adjusted volume (i.e., $65 \mathrm{~dB}$ above participant's average hearing threshold between 250 and $3000 \mathrm{~Hz}$ as assessed by means of pure-tone audiometry; compare Passow et al., 2014). All 12 dichotic syllable pairs were presented six times in each of the attention and arousal conditions, summing to 288 trials in total (i.e., 12 syllable pairs $\times 6$ presentations $\times 2$ attentional focus $\times 2$ arousal conditions) that were split in blocks of 48 trials. In $8.33 \%$ of the trials, no CS $\left(\mathrm{CS}^{+} ; \mathrm{CS}^{-}\right)$ was displayed (no-CS trials; $\mathrm{n}=24$ ), with a fixation cross instead occurring at that time point to obtain an index of auditory attention regardless of CS presentation. In another $8.33 \%$ of the trials, the $\mathrm{CS}^{+}$was followed by an electrical shock (booster trials; compare fear conditioning phase; $n=24$; compare Lee et al., 2018) to prevent extinction of the conditioned response. No-CS and booster trials were excluded from analyses. After each block, participants had a self-paced break during which their average accuracy was displayed graphically. Breaks were followed by a brief reconditioning period of 20 trials that resembled one-half of the fear conditioning phase (presented in pseudorandomized order) to maintain the fear response throughout the experiment (Fig. 1c).

To thoroughly familiarize participants with both the auditory material and the instructions before testing, on the first assessment day, we presented the six syllables first diotically (i.e., the same syllable at the same time to the left and right ear; 24 trials) followed by a presentation to only one ear (6 left and 6 right ear trials). Participants indicated by button press which syllable they heard/on which ear (mean \pm SEM accuracy: $94.801 \pm 0.983 \%)$. In addition, participants completed a dichotic listening task without arousal manipulation (96 trials; accuracy: $46.749 \pm 0.010 \%$; chance performance in this task is $33.3 \%$, i.e., one of three possible choices).

All stimuli were presented using Psychtoolbox (Psychophysics Toolbox; RRID:SCR_002881) for MATLAB (The MathWorks; RRID: SCR_001622) and insert earphones (ER 3A; Etymotic Research). During the fear conditioning and dichotic listening task, gaze position, pupil dilation, and the EEG (day 2 only) were continuously recorded (see below). To minimize the influence of sensory input on pupil dilation, testing took place in a dark, sound-attenuated, and electromagnetically shielded room (compare Hong et al., 2014). Further, to minimize the influence of eye movements on pupil dilation (Gagl et al., 2011) at the beginning of each trial (baseline period) participant's gaze position was sampled online and the trial only started if central fixation was either maintained $(>75 \%$ of the time) or restored on presentation of a re-fixation target.

\section{Behavioral analyses}

Dichotic listening task. To evaluate age differences in auditory selective attention, we calculated the auditory laterality index (LI; Marshall et al., 1975), for each attentional focus condition (FL, FR), collapsing across arousal conditions. This index expresses the amount of right relative to left ear responses (i.e., $\mathrm{LI}=[$ right $-\mathrm{left}] /[$ right + left $]$ ). The $\mathrm{LI}$ ranges from -1 to 1 , whereby negative values indicate more left ear responses and positive values index a tendency toward selecting the right ear syllable. The Younger and older adults' LIs were analyzed in a two-factorial (age group $\times$ attentional focus [FL, FR]) mixed-measures ANOVA that was followed-up by post hoc tests within age groups. To judge the influence of age-related hearing loss on auditory-selective attention, in a second ANOVA (age group $\times$ attentional focus), we included participants' average hearing loss as a covariate. For further analyses, the difference between LIs for the FL and FR condition was calculated to provide an overall measure of participants' auditory-selective attention ability.

General attention. To obtain a single measure for general attention performance (i.e., independent of the specific task used), we made use of the comprehensive cognitive battery available for this dataset. In particular, we integrated performance across multiple visual and auditory attention tasks (i.e., dichotic listening task; D2 test of attention; DSST; auditory DST) by means of a structural equation modeling approach (see Fig. 10, bottom) using the $\Omega$ nyx 1.0-1013 software package (von Oertzen et al., 2015) with full information maximum likelihood estimation. Structural equation modeling offers a multivariate approach in which observed (manifest) variables can be used to examine hypotheses about unobserved (latent) variables. Latent variables have the benefit of accounting for measurement error in observed scores and thereby increasing statistical power (Curran et al., 2010; Kievit et al., 2018).

In particular, in each age group, standardized performance in the dichotic listening task, D2 test of attention, DSST, and DST served as manifest variables and loaded on a single latent selective attention factor (i.e., a multiple-group model). Factor loadings (other than the first, which was fixed to 1) were estimated freely but were constrained to be equal across groups. The model demonstrated metric factorial invariance (i.e., it required variant manifest intercepts and variances across groups) and thus precluded an interpretation of age group differences in the means of the latent factor (Meredith and Teresi, 2006; Schwab and Helm, 2015). We assessed the adequacy of the proposed selective attention model by testing for differences between the model-implied and empirically observed covariance matrices (Eid et al., 2015). The $\chi^{2}$ test formally tests for equity of the covariance matrices. However, since it is particularly sensitive to sample size, it should be interpreted with caution in large samples (Brown, 2006; Eid et al., 2015). We thus additionally examined two frequently reported fit indices: (1) the root mean square error of approximation (RMSEA), which is a closeness-of-fit coefficient expressing how much the postulated model approaches the true model; and (2) the comparative fit index (CFI), an incremental fit index, which compares the goodness of fit of the proposed model with a more restrictive nested baseline model (Brown, 2006; Curran et al., 2010; Eid et al., 2015). In contrast to the $\chi^{2}$ test, the RMSEA and CFI are not influenced by sample size. RMSEA values close to $<0.06$ and CFI values of close to $\geq 0.95$ indicate good model fit (Brown, 2006). After establishing model fit, differences in parameters of interest were tested by fixing parameters to zero and comparing model fit to a model in which parameters were freely estimated using a likelihood ratio difference test (Curran et al., 2010; Eid et al., 2015).

\section{Physiologic data recording and preprocessing}

Pupil dilation. We recorded participants' pupil dilation as a proxy for central LC-NE activity (Reimer et al., 2014; Costa and Rudebeck, 2016; Joshi et al., 2016; Breton-Provencher and Sur, 2019; Deitcher et al., 2019; Zerbi et al., 2019) along with gaze position using an infrared video-based 
eye tracker (EyeLink 1000 desktop mount; monocular setup; SR Research) with a spatial resolution of up to $0.25^{\circ}$ and a sampling rate of $1000 \mathrm{~Hz}$. A forehead and chin rest $53.5 \mathrm{~cm}$ from the computer screen was used to minimize participants' head movements during measurements. Participants were instructed to maintain central fixation throughout all experiments, and compliance with this instruction was enforced at the beginning of every trial. Each experiment started with a (re)calibration of the eye-tracking system using a standard 5 point grid. During (re)calibration, fixation errors were kept $<0.5^{\circ}$.

For synchronous, integrated analysis of eye-tracking and EEG data, we used the Eye-EEG toolbox (Dimigen et al., 2011), an extension for the open-source MATLAB toolbox EEGLab (RRID:SCR_007292; Delorme and Makeig, 2004) as well as the FieldTrip toolbox (RRID:SCR_004849; Oostenveld et al., 2011). Eye-tracking data of the re/conditioning and dichotic listening sessions were resampled to $500 \mathrm{~Hz}$ and segmented in bins of $8.5 \mathrm{~s}$ (i.e., -1.5 to $7 \mathrm{~s}$ with respect to CS onset). Time segments contaminated by blinks or excessive eye movements were automatically detected and imputed using custom-written MATLAB code. In particular, segments falling $>3$ SDs below a participant's median pupil dilation (calculated across the whole experiment) were considered as blink or partly occluded pupil. Further, periods with excessive eye movements, as indicated by $z$-scored vertical gaze channel values $>3$ (computed across the whole experiment), were considered artifacts. All artifacts were padded by \pm 50 samples to account for biased pupil estimates shortly before/after artifacts (compare de Gee et al., 2014).

Excluding detected artifacts, we computed the average event-related pupil response (ERPR) for each trial category (i.e., $\mathrm{CS}^{+}$; $\mathrm{CS}^{-}$; separately for fear re/conditioning and dichotic listening data; Fig. 1c). In all trials, artifact-containing segments were then replaced by the corresponding time segments of the demeaned average response centered at the given trial. Because of technical issues, no pupil data were available for one YA for fear conditioning on day 2 and one OA for fear conditioning on day 3 and the dichotic listening task (day 2).

Notably, we performed a set of control analyses that included a linear interpolation of missing pupil samples (compare de Gee et al., 2014) instead of the mean imputation approach described above. Importantly, regardless of the preprocessing pipeline, qualitatively similar results are obtained on the group level.

EEG. To evaluate neural responses during re/conditioning and dichotic listening (compare Fig. 1c), we recorded the EEG. Data were continuously sampled from $61 \mathrm{Ag} / \mathrm{AgCl}$ electrodes embedded in an elastic cap that were placed according to the 10-10 system using BrainVision Recorder (BrainAmp DC amplifiers, Brain Products, Braincap, and BrainVision, respectively). An electrode above the forehead (AFz) served as ground. Three additional electrodes were placed next to each eye and below the left eye to acquire horizontal and vertical electro-oculograms. Data were sampled at $1000 \mathrm{~Hz}$ in a bandwidth between 0.1 and $250 \mathrm{~Hz}$ and online-referenced to the right mastoid while the left mastoid was recorded as an additional channel. During EEG preparation, electrode impedances were kept $<5 \mathrm{k} \Omega$.

EEG data processing was performed by means of the Eye-EEG (Dimigen et al., 2011), EEGLab (Delorme and Makeig, 2004), and FieldTrip (Oostenveld et al., 2011) toolboxes in addition to custom-written MATLAB code. For analyses, data were demeaned, rereferenced to mathematically linked mastoids, downsampled to $500 \mathrm{~Hz}$, and bandpass-filtered $(0.2-125 \mathrm{~Hz}$; fourth-order Butterworth). A multistep procedure was applied to purge data of artifacts: First, data were visually screened for periods of excessive muscle activity; and subsequently, independent component analysis was used to identify and remove components related to eye, muscle, and cardiac activity (e.g., Jung et al., 2000). Next, data were segmented in $8.5 \mathrm{~s}$ epochs $(-1.5$ and $7 \mathrm{~s}$ with respect to stimulus onset) and submitted to a fully automatic thresholding approach for artifact rejection (compare Nolan et al., 2010). Excluded channels were interpolated with spherical splines (Perrin et al., 1989). Finally, remaining trials were again visually screened to determine successful cleaning. During preprocessing, on average, $36.271 \pm 1.667 \%$ of trials were excluded from further analyses.

Time-varying power information for each trial and electrode was then extracted by convolution of the cleaned time domain signal with a series of Morlet wavelets with a length of seven cycles (compare Herrmann et al., 2005; Werkle-Bergner et al., 2009). Time-varying power estimates were computed for frequencies between 1 and $40 \mathrm{~Hz}$ (in steps of $1 \mathrm{~Hz}$ ) in a time window between -1.5 to $7 \mathrm{~s}$ with respect to stimulus onset (time bins of $4 \mathrm{~ms}$ ), separately for $\mathrm{CS}^{+}$and $\mathrm{CS}^{-}$trials of the reconditioning and dichotic listening phases (Fig. 1c).

\section{Physiologic data analyses}

Within modality within-subject statistics (first level). Within younger and older subjects, we contrasted arousing $\left(\mathrm{CS}^{+}\right)$and neutral control trials $\left(\mathrm{CS}^{-}\right)$by means of independent-samples $t$ tests to isolate arousal-associated response patterns. Contrasts were computed for time domain pupil data (i.e., ERPR), time domain EEG data (i.e., ERP), and time-frequency domain EEG data (i.e., event-related desynchronization [ERD]). To counteract potential unequal distribution of $\mathrm{CS}^{+}$and $\mathrm{CS}^{-}$trials (e.g., more artifacts in arousing trials), we iteratively selected random, equal-sized subsets of the available trials using a bootstrapping procedure $\left(n_{\text {bootstraps }}=50\right.$ iterations; $n_{\text {SelectedTrials }}=$ lowest trial number across conditions -1$)$. The mean $t$ value over the 50 bootstraps served as final first-level test statistic that was passed on to the second level (see below). First-level statistics were computed within subjects for conditioning (separately for each day [days 1-3]; Fig. 1c), reconditioning, and dichotic listening trials. While the contrast $\left(\mathrm{CS}^{+}\right.$vs $\left.\mathrm{CS}^{-}\right)$remained the same across these analyses, during re/ conditioning, participants received electrical stimulation (US); thus, the observed responses may represent a mixture of fear and somatosensory responses. In contrast, during the dichotic listening task, no shocks were applied; thus, observed arousal responses $\left(\mathrm{CS}^{+}\right.$vs $\left.\mathrm{CS}^{-}\right)$indicate the reinstatement of the fear response (compare Fig. 1c).

Notably, the $\mathrm{CS}^{+}$versus $\mathrm{CS}^{-}$first-level $t$ maps express the difference in pupil and EEG responses to arousing and nonarousing stimuli. That is, in the strict sense of the word, they do not constitute an ERPR/ERP/ ERD, but rather the standardized difference between two pupil/EEG responses. However, to ease readability, we use the terms ERPR, ERP, and ERD also for these contrasts.

Within-modality group statistics (second level). For analyses on the group level, we contrasted first-level $t$ maps (i.e., $\mathrm{CS}^{+}$vs $\mathrm{CS}^{-}$) against zero to identify neural correlates associated with the arousal manipulation in conditioning and reconditioning trials (compare Fig. 1c) that were shared across subjects in each group. Analyses were run separately for each day, first across all subjects (YA and OA) and then within YA and OA for all modalities (ERPR, ERP, and ERD data). In particular, we calculated nonparametric, cluster-based, random permutation tests as implemented in the FieldTrip toolbox that effectively control the false alarm rate in case of multiple testing (Maris and Oostenveld, 2007; Oostenveld et al., 2011). The same statistical procedure was applied to $2 \mathrm{D}$ (i.e., channel $\times$ time) and $3 \mathrm{D}$ (i.e., channel $\times$ frequency $\times$ time) data. That is, ERPR, ERP, and ERD were analyzed in the same manner. Here, however, only the approach for 3D data (ERD) is described to ease readability. In short, first, a two-sided, dependent-samples $t$ test was calculated for each spatio-spectral-temporal (channel $\times$ frequency $\times$ time) sample. Neighboring samples with a $p$ value $<0.05$ were grouped with spatially, spectrally, and temporally adjacent samples to form a cluster. The sum of all $t$ values within a cluster formed the respective test statistic $\left(t_{\text {sum }}\right)$. A reference distribution for the summed cluster-level $t$ values was computed via the Monte Carlo method. Specifically, in each of 1000 repetitions, group membership was randomly assigned, a $t$ test computed, and the $t$ value summed for each cluster. Observed clusters whose test statistic exceeded the 97.5th percentile for its respective reference probability distribution were considered significant.

On a group level, cluster statistics revealed reliable arousal effects during conditioning and reconditioning within all modalities (ERPR, ERP, and ERD). To evaluate a potential reinstatement of these fear responses also during the dichotic listening task (in which no shocks were applied anymore; compare Fig. $1 c$ ), each subject's first-level $\left(\mathrm{CS}^{+}\right.$ vs $\mathrm{CS}^{-}$) dichotic listening data were averaged across spatio-spectral-temporal samples, which reached significance on a group level during the reconditioning period. That is, we applied the observed reconditioning fear response (i.e., significant cluster) as a search space to evaluate its reinstatement within the dichotic listening data. This approach yielded a 
single reinstatement value for each subject for each modality (i.e., ERPR, ERP, and ERD data). Within modalities, the reliability of the reinstatement was then determined by means of nonparametric Wilcoxon signed rank $(W)$ tests (across and within age groups).

To judge the temporal stability of fear-conditioned pupil responses (ERPR) over assessment days (days 1-3; compare Fig. 1c), in addition, each subject's first-level $\left(\mathrm{CS}^{+}\right.$vs $\left.\mathrm{CS}^{-}\right)$conditioning and reconditioning ERPR data were averaged across time points, which reached significance on a group level (i.e., second-level statistic for the respective day). This yielded a single ERPR value for each subject for each conditioning session (days 1-3) and the reconditioning phase (day 2). We then used intraclass correlations (two-way mixed; consistency) to evaluate the temporal stability of participants' fear-conditioned pupil dilation.

Cross-modality group statistics. To determine whether EEG correlates of the arousal manipulation (i.e., ERP, ERD) were linked to the LC-NE system, we correlated participants' EEG responses with their pupil dilation, a proxy of noradrenergic activity, across age groups. We assessed this association within the dichotic listening data, since this provides an estimate of the reinstatement of the fear response regardless of potential somatosensory artifacts related to the reinforcement (US; Fig. 1). In particular, participants' first-level EEG $t$ maps $\left(\mathrm{CS}^{+}\right.$vs $\left.\mathrm{CS}^{-}\right)$were correlated with participants' average pupil reinstatement (see within modality group statistics) in a nonparametric, cluster-based, random permutation framework as implemented in the FieldTrip toolbox. Analyses were run separately for ERP and ERD data; however, here only the approach for time-frequency data (ERD) is described to ease readability. For each spatio-spectral-temporal sample, a two-sided Pearson's correlation between the EEG and the pupil reinstatement data were calculated. As done for the within-modality statistics, neighboring samples with a $p$ value $<0.05$ were grouped with spatially, spectrally, and temporally adjacent samples to form a cluster. The sum of all $\rho$ values within a cluster formed the respective test statistic. A reference distribution for the summed cluster-level $\rho$ values was computed via the Monte Carlo method. In particular, the null hypothesis of statistical independence between EEG and pupil data was tested by randomly permuting pupil estimates between subjects over 1000 repetitions. For each repetition, a correlation was computed, and $\rho$ values were summed for each cluster. Observed clusters whose test statistic exceeded the 97.5th percentile for its respective reference probability distribution were considered significant. To specifically target reinstatement responses, we restricted the cross-modality analyses to EEG samples that showed a reliable arousal effect (i.e., were part of significant clusters in the second-level reconditioning analyses). However, analyses were performed solely on reinstatement data (i.e., dichotic listening ERPR, ERP, and ERD data; compare Fig. 1).

Cross-modality structural equation model. Cluster-correlation analyses revealed reliable associations between EEG and pupil reinstatement for both ERP and ERD data, suggesting a common underlying pupil-EEG factor. For each subject, we thus extracted and averaged those samples of the EEG reinstatement response that showed a reliable link to pupil reinstatement. This returned a single pupil-associated reinstatement estimate for ERP and ERD data, respectively. As for the behavioral data, we then used a structural equation modeling approach to integrate over the interrelated indicators of the arousal response (see Fig. 10, top). In particular, in YA and OA, standardized pupil reinstatement and pupil-associated EEG reinstatement estimates served as manifest variables and loaded on a single, latent $\mathrm{NE}$ responsiveness factor (i.e., a multiple-group model). Factor loadings (other than the first, which was fixed to 1) were estimated freely but were constrained to be equal across groups. The model demonstrated strict factorial invariance (i.e., showed invariant manifest intercepts and variances across groups) and thus allowed an interpretation of age group differences in the means of the latent factor (Meredith and Teresi, 2006; Schwab and Helm, 2015). We evaluated age differences in latent NE responsiveness by means of Spearman correlations (across age groups). Adequacy of the proposed model was assessed using a $\chi^{2}$ test as well as two additional fit indices (RMSEA, CFI; see above).

Analyses of associations between physiological and behavioral data After generating structural equation models for our cognitive and physiological measures, respectively, we set out to link both modalities. That is, we were interested in assessing the relation between interindividual

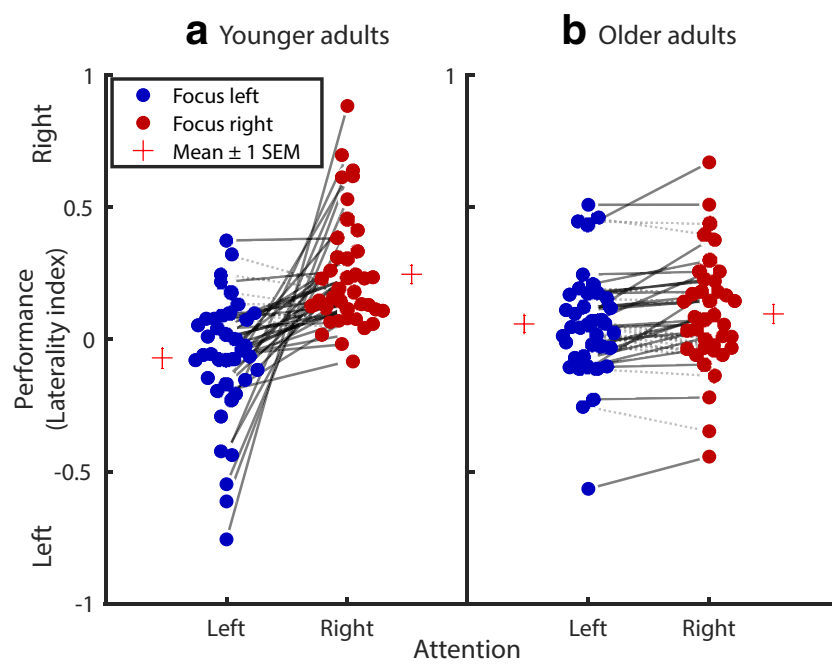

Figure 2. Selective auditory attention performance of $Y A(\boldsymbol{a})$ and $O A(\boldsymbol{b})$ in a dichotic listening task. Negative and positive LI values indicate a tendency for left and right ear responses, respectively. Circles connected by solid lines represent participants who demonstrate a behavioral selective attention effect (i.e., more responses of the cued ear relative to the not cued ear), with the slope of the lines reflecting the degrees of attentional modulation. Circles connected by gray dotted lines represent a reversed effect. While the amount of selective attention is markedly decreased in $\mathrm{OA}$, both younger and older participants demonstrate reliable selective attention on a group level. $\mathrm{LI}=(\mathrm{FR}-\mathrm{FL}) /(\mathrm{FR}+\mathrm{FL}))$.

Table 2. Overview of age differences in attention ${ }^{a}$

\begin{tabular}{llllll}
\hline Task & $N($ YA/OA $)$ & Performance & Performance & & \\
\hline DLT $^{b}$ & $77(3 \pm$ SEM $)$ & OA $(z \pm$ SEM $)$ & $z$ & $p$ \\
D2 & $77(39 / 38)$ & $0.415(0.200)$ & $-0.425(0.048)$ & 3.790 & $<0.001$ \\
DSST & $77(39 / 38)$ & $0.634(0.133)$ & $-0.651(0.113)$ & 6.109 & $<0.001$ \\
DST & $77(39 / 38)$ & $0.206(0.129)$ & $-0.645(0.120)$ & 5.689 & $<0.001$ \\
\hline
\end{tabular}

${ }^{a}$ For each age group, for all cognitive tasks, mean performance is provided as $z$ value \pm SEM. DLT, Dichotic Listening Task; D2, D2 test of attention. All comparisons are evaluated using nonparametric Mann-Whitney $U$ tests (YA vs $O A$ ).

${ }^{b}$ The difference between LIs for the FL and FR condition was calculated to provide an overall measure of participants' auditory-selective attention ability.

differences in attention and interindividual differences in NE responsiveness. For this, we first built a unified model merging the attention and responsiveness models described above (see Fig. 10). We then investigated associations between cognitive and physiological factors by allowing for freely estimated covariances on a latent level (see Fig. 10, yellow). As before, model fit for all described models was determined using a $\chi^{2}$ test in combination with two additional fit indices (RMSEA, CFI).

\section{Control analyses}

To additionally explore the behavioral relevance of moment-to-moment fluctuations in arousal, we conducted mixed-effects logistic regression analyses (i.e., a generalized linear mixed-effects model). We used maximum likelihood with Laplace approximation to estimate model parameters as implemented in MATLAB's statistics and machine learning toolbox.

First, to quantify the relative arousal level for each trial, physiological data were standardized by calculating the distance to a reference distribution consisting of all $\mathrm{CS}^{-}$trials using one-sample $t$ tests (for each sample within a trial) as follows:

$$
\begin{gathered}
\text { standardized trial }=\left(\text { mean }\left(\text { all CS }{ }^{-} \text {trials }\right)-\text { current trial }\right) / \\
\text { standard deviation }\left(\text { all CS }{ }^{-} \text {trials }\right)
\end{gathered}
$$

Next, we extracted and averaged those samples of each trial's standardized pupil and EEG (ERP, ERD) response that showed a reliable 
a

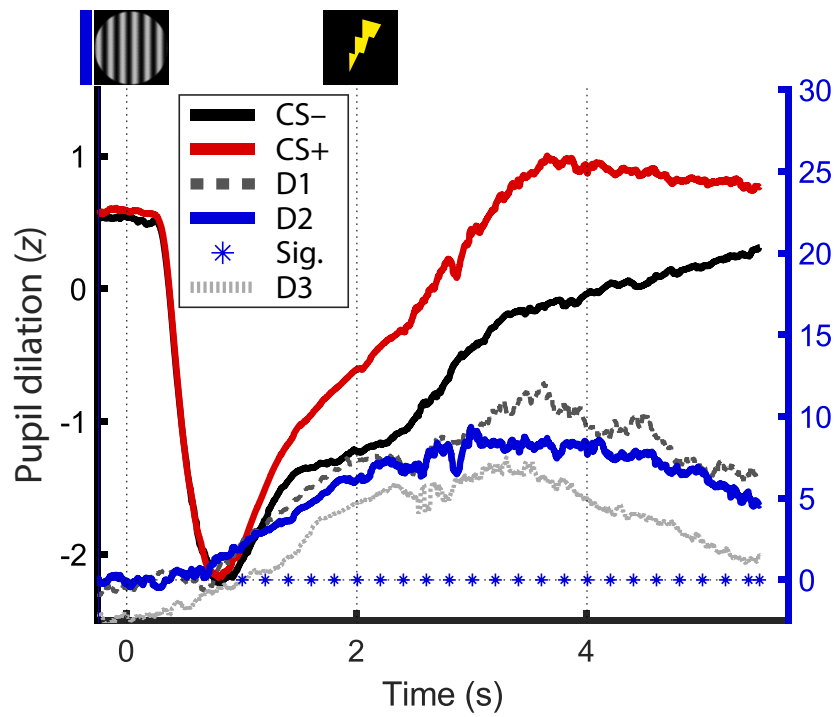

b

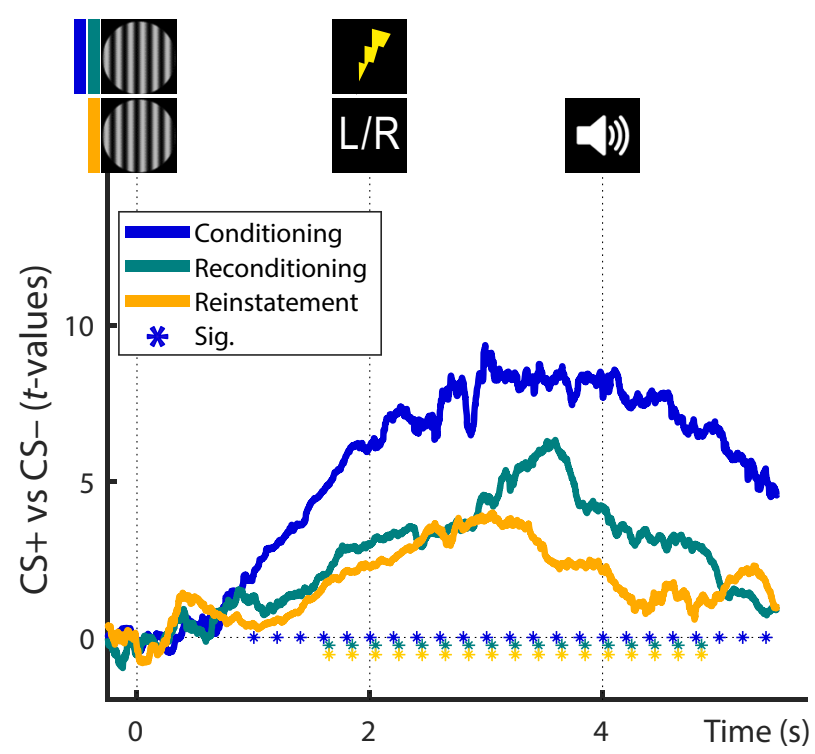

\section{Older adults}

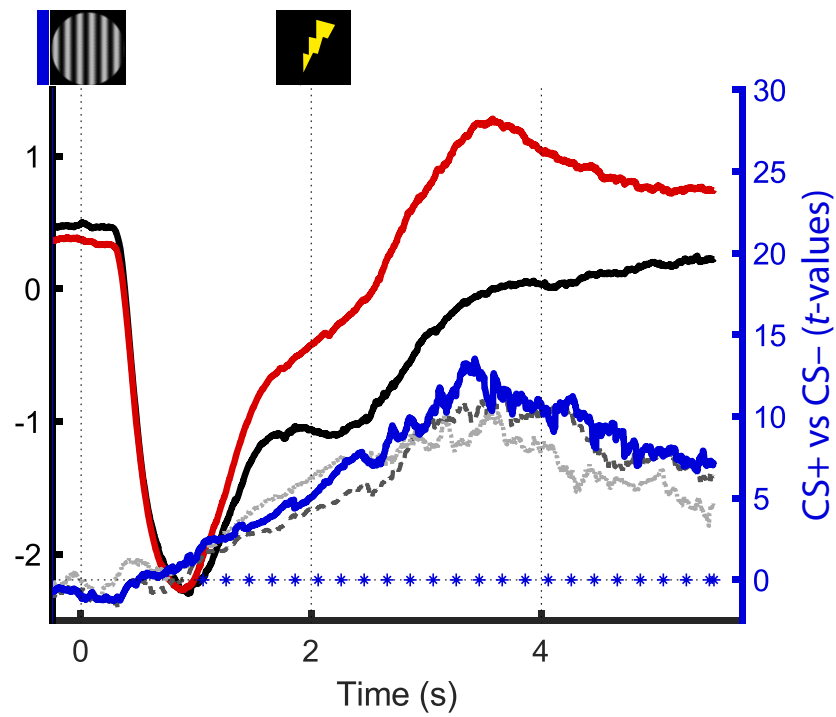

\section{Older adults}

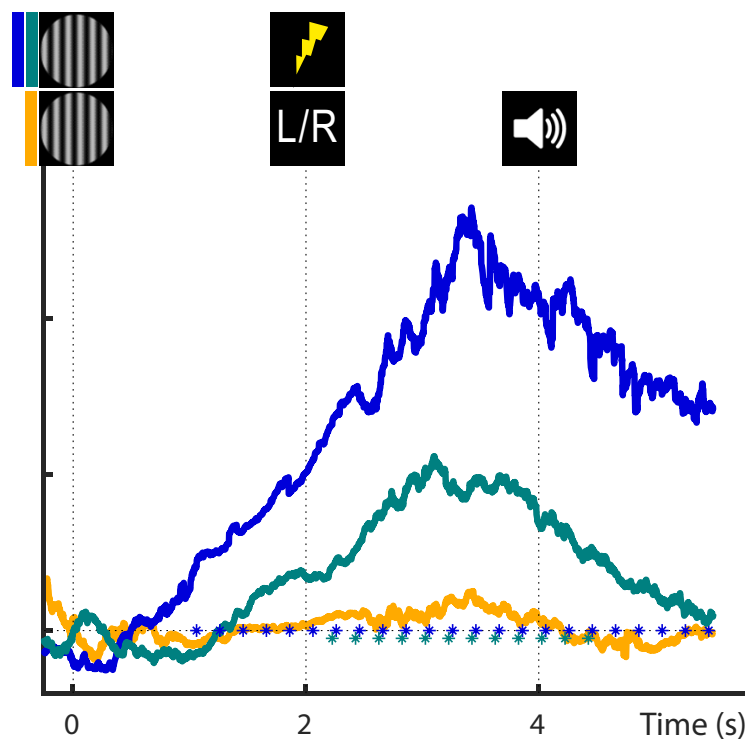

Figure 3. Average pupil dilation of YA and $\mathrm{OA}$ in response to the presentation of fear-conditioned (CS ${ }^{+}$; red) and neutral control stimuli (CS- ; black) during fear conditioning $(\boldsymbol{a}$; day 2$)$ and during the dichotic listening task ( $\boldsymbol{b}$; i.e., reconditioning and reinstatement). Group statistics depict the consistency of the $\mathrm{CS}^{+}$versus $\mathrm{CS}^{-}$contrast on the second level. $\boldsymbol{a}$, Statistics are presented for conditioning data for the first (D1; light gray, dashed), second (D2; solid, blue), and third (D3; dark gray, dotted) day of conditioning assessments (see right $y$ axis). $\boldsymbol{b}$, Pupil responses during conditioning (blue), reconditioning (teal), and dichotic listening trials (reinstatement; orange) on day 2. Horizontal lines of asterisks indicate significant time windows (blue). Reinstatement of the fear-conditioned pupil response during the dichotic listening task (see Fig. 1c) is evaluated statistically using Wilcoxon tests (YA: $W(39)=586 ; Z=2.735 ; p=0.006 ; 0 \mathrm{~A}: W(37)=364$; $Z=0.189 ; p=0.850$ ). For visualization, averaged pupil traces (black and red lines) are standardized ( $z$-scored).

arousal effect on the group level (see Figs. 3, 5, and 7). In addition to these poststimulus predictors, we also included a prestimulus baseline factor $(-1$ to $0 \mathrm{~s})$ for the pupil and EEG data. For the EEG data, baseline samples were restricted to those electrodes and frequencies that were part of the poststimulus cluster.

The extracted physiological data were first used to predict each trial's accuracy (target/distractor selected, i.e., a binomially distributed response). Outlier trials with $z$ scores $>3$ or $<-3$ were excluded from the analyses. Further, trials in which no response was given or a novel, not presented syllable was picked were also dropped (overall, $12.226 \%$ of all trials were excluded, leaving 13,331 trials for analyses).

In addition to physiological factors, age group and trial condition (CS type $\left[\mathrm{CS}^{+}, \mathrm{CS}^{-}\right]$, attentional focus condition [FL, FR]) were added as additional fixed effects. Beyond main effects of physiological factors and trial condition, we allowed for interactions between arousal condition (i.e., $\mathrm{CS}^{+}, \mathrm{CS}^{-}$) and the phasic physiological responses. Individual performance differences were modeled as random effects of intercept grouped by individual participant (ID). The model formula in Wilkinson notation is expressed as follows: 


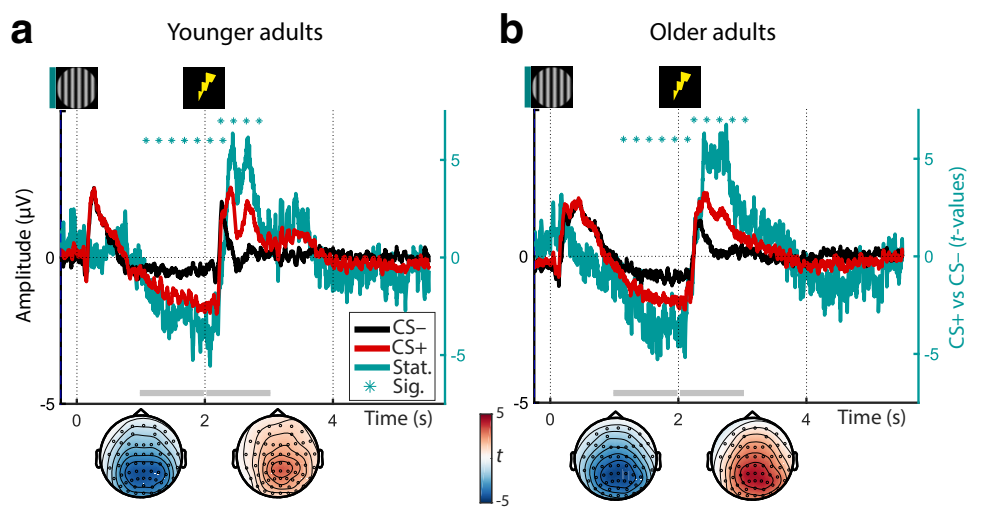

Figure 4. Averaged ERPs of $\mathrm{YA}(\boldsymbol{a})$ and $\mathrm{OA}(\boldsymbol{b})$ in response to the presentation of fear-conditioned (CS ${ }^{+}$; red) and neutral control stimuli (CS ; black) during reconditioning (left $y$ axis; on day 2). Group statistics depict the consistency of the $\mathrm{CS}^{+}$vs. $\mathrm{CS}^{-}$contrast on the second level (teal) and are shown on the right $y$ axis. Horizontal lines of asterisks indicate significant time windows. The topography of the group statistics between 1-2 and 2$3 \mathrm{~s}$ relative to $\mathrm{CS}$ onset is shown below the time courses (gray horizontal bars). For visualization, time courses are averaged across all electrodes.

$$
\begin{gathered}
\operatorname{logit}(\text { accuracy }) \sim 1+\text { CS }+ \text { attentional_condition }+ \text { age_group } \\
+ \text { ERP + ERP_baseline + pupil + pupil_baseline } \\
+ \text { ERD + ERD_baseline + CS }: \text { ERP + CS : pupil } \\
+ \text { CS }: \text { ERD }+(1 \mid \text { ID })
\end{gathered}
$$

Finally, we probed whether the pupil-EEG associations tested on the group level (see Cross-modality group statistics) would also hold within subjects on a single-trial level. To this end, we again calculated mixedeffects logistic regression analyses as described above. However, this time, instead of accuracy, we used phasic pupil dilation as (continuous) dependent variable as follows:

$$
\begin{array}{r}
\operatorname{logit}(\text { pupil }) \sim 1+\text { CS }+ \text { age_group }+ \text { pupil_baseline } \\
+ \text { ERP + ERP_baseline }+ \text { ERD }+ \text { ERD_baseline }+(1 \mid \text { ID })
\end{array}
$$

\section{Code and data availability}

The custom code and preprocessed data used for the analyses are available on an Open Science Framework repository (https://osf.io/g9fqj/).

\section{Results}

\section{Impaired selective attention in older adults}

Participants demonstrated successful auditory selective attention in the dichotic listening task as indicated by a two-factorial mixed-measures ANOVA (age group $\times$ attentional focus; main effect of attentional focus: $\left.F_{(1,75)}=26.413, p<0.001, \eta^{2}=0.260\right)$. Post hoc analyses within YA and OA demonstrated that both groups were able to exert auditory-selective attention (one-factorial repeated measures ANOVA; main effect of attentional focus: for YA $F_{(1,38)}=22.803, p<0.001, \eta^{2}=0.375$; for OA $F_{(1,37)}=$ 5.702, $\left.p=0.022, \eta^{2}=0.134\right)$.

YA and OA, however, differed reliably in their ability to modulate their attentional focus. While the age group main effect was not significant $\left(F_{(1,75)}=0.087, p=0.769, \eta^{2}=0.001\right)$, we observed a reliable age group $\times$ attentional focus interaction $\left(F_{(1,75)}=16.318, p<0.001, \eta^{2}=0.179\right.$; Fig. 2$)$, indicating impaired selective attention in old age. Here a main effect of age (e.g., lower LI in OA compared with YA) would indicate better performance in one attentional focus condition (e.g., FL) and worse performance in the other (e.g., FR; compare Fig. 2). The observed age group $\times$ attentional focus interaction in contrast reveals worse performance in $\mathrm{OA}$ in both conditions (i.e., lower LI values in the FR condition [YA: $0.247 \pm 0.035$; OA: $0.097 \pm 0.036]$; higher LI values in the FL condition [YA: $-0.070 \pm 0.039$; OA: $0.058 \pm 0.033$ ]; Table 2; Fig. 2).

Post hoc analyses indicated that age differences in auditory selective attention were not explained by age-related differences in hearing loss (i.e., age group $\times$ attentional focus mixed-measures ANOVA, including hearing loss as covariate: age group $\times$ attentional focus: $F_{(1,74)}=4.862, p=0.031$, $\eta^{2}=0.062$; hearing loss $\times$ attentional focus: $F_{(1,74)}$ $\left.=0.740, p=0.393, \eta^{2}=0.010\right)$. Similarly, control analyses indicated that age differences in auditory selective attention persisted after including interaural threshold differences in the model (i.e., age group $\times$ attentional focus mixed-measures ANOVA, including interaural threshold differences as covariate: age group $\times$ attentional focus: $F_{(1,71)}=16.143, p<$ $0.001, \eta^{2}=0.185$; interaural threshold $\times$ attentional focus: $\left.F_{(1,71)}=0.462, p=0.499, \eta^{2}=0.006\right)$.

We replicated this finding of impaired selective attention in aging across multiple visual and auditory attention tasks using nonparametric Mann-Whitney $U$ tests (all $p<0.05$; Table 2). In order to later reliably relate attention performance to physiological indices of the LC-NE system (see below), we integrated performance over tasks to derive a single measure reflecting general attention performance (Fig. 10, bottom). The proposed model fit the data well $\left(\chi^{2}=9.827, \mathrm{df}=23\right.$; $\mathrm{RMSEA}=0.0 ; \mathrm{CFI}=1.594$; Brown, 2006). The variances of the attention factors differed reliably from zero in both age group (all $\Delta \chi^{2} \geq 11.225, \Delta \mathrm{df}=1$, all $p<0.001$ ), indicating interindividual differences in attention.

\section{Stable fear-conditioned pupil dilation in younger adults and older adults}

In the conditioning and reconditioning phases of the assessment (see Fig. 1c), YA and OA demonstrated a reliable, multimodal response to the arousal manipulation. In the following, first modality-specific results are reported (i.e., pupil dilation and EEG) before detailing their interrelation.

During fear conditioning and reconditioning, conditioned stimuli $\left(\mathrm{CS}^{+}\right.$vs $\left.\mathrm{CS}^{-}\right)$reliably elicited pupil dilation over prolonged time windows as revealed by cluster permutation analyses (both across and within age groups; all $p_{\text {corr }}<0.01$; Fig. 3 ).

Fear-conditioned pupil responses demonstrated a moderate to high stability across assessments (days 1-3) as indicated by intraclass correlations (two-way mixed; consistency; intraclass correlation $[95 \% \mathrm{CI}]=0.652[0.502-0.766] ; p<0.001)$. In line with pupil dilation as a noninvasive marker of LC activity, this points to a stable phasic activation of the NE system by fear-conditioned stimuli (and US) across age groups.

\section{Reinstatement of pupil dilation in younger adults}

In the absence of reinforcements (US), fear-conditioned stimuli maintained their arousing nature and led to a marginally significant reinstatement of pupil dilation across groups $(W(76)=$ 1839; $Z=1.948 ; p=0.052$ ). While YA demonstrated a robust reinstatement effect, in OA reinstatement did not reach statistical significance on a group level (Fig. 3; YA: $W(39)=586 ; Z=2.735$; $p=0.006$; OA: $W(37)=364 ; Z=0.189 ; p=0.850)$. The lack of pupil reinstatement in $\mathrm{OA}$ presumably reflects age-related difficulties in triggering and maintaining self-initiated processing (i.e., 

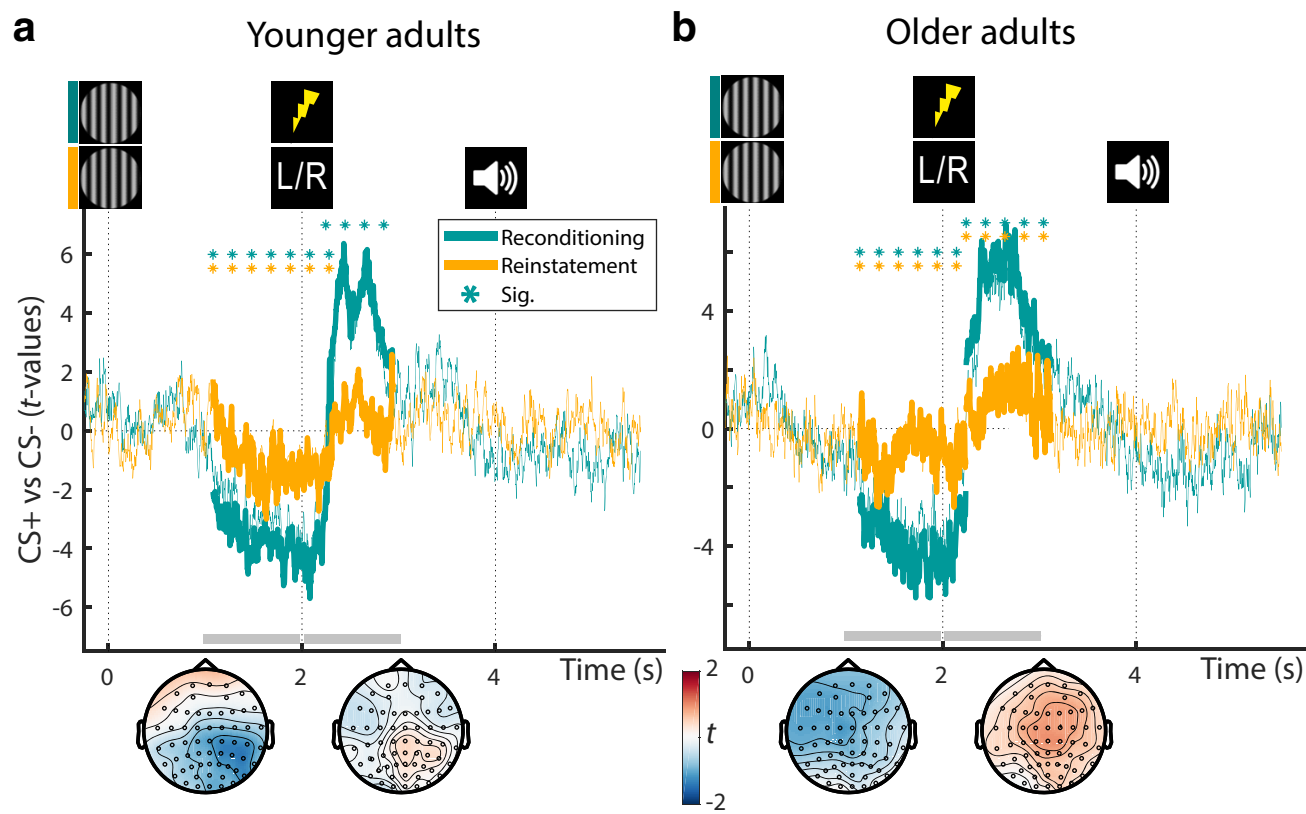

Figure 5. Group statistics for YA (a) and OA (b): ERPs during reconditioning (teal; compare Fig. 4) and dichotic listening trials (orange; reinstatement) on day 2. All statistics depict the consistency of the $\mathrm{CS}^{+}$versus $\mathrm{CS}^{-}$contrast on the second level. Teal horizontal lines of asterisks indicate the extent of the significant reconditioning clusters. Reinstatement of the earlier, negative, and later, positive, ERP clusters during the dichotic listening task is evaluated statistically using Wilcoxon tests (see orange lines of asterisks; YA: negative: $W(39)=214 ; Z=-2.456 ; p=0.014$; positive: $W(39)=456 ; Z=0.921 ; p=0.357 ; 0$ A: negative: $W(38)=214 ; Z=-2.270 ; p=0.023$; positive: $W(38)=582 ; Z=3.067 ; p=0.002$ ). The topography of the reinstatement group statistics between $1-2$ and $2-3$ s relative to $C S$ onset is shown below the time courses (gray horizontal bars). $\mathrm{CS}^{+}=$fear conditioned stimulus; $\mathrm{CS}^{-}=$perceptually matched, neutral control stimulus. For visualization, time courses are averaged across all electrodes.

reinstatement; Lindenberger and Mayr, 2014) in line with previous reports (van Gerven et al., 2004). By contrast, age differences are known to be reduced or even disappear when OA can rely on external information (e.g., reminders), such as the reinforcements (US) during re/conditioning. The age difference in the reinstatement of pupil dilation approached statistical significance (YA vs OA: $U(76)=1671 ; Z=1.756 ; p=0.079$ ). Since the reinstatement of pupil dilation occurs in the absence of somatosensory stimulation and associated artifacts, it is attributed to the arousal response following the reactivation of the fear memory. We thus interpret the reinstatement of the fear-induced pupil response as indicator of the effectiveness of the LC-NE system in modulating memory, which trends to be reduced in aging.

Fear-conditioned parietal ERPs in younger adults and older adults

During fear reconditioning, conditioned stimuli also reliably elicited event-related EEG responses (ERP) both across and within age groups as revealed by cluster permutation analyses (all $p_{\text {corr }}<$ 0.01 ; Fig. 4). In particular, we observed that after an initially similar ERP $\left(<1\right.$ s) to $\mathrm{CS}^{+}$and $\mathrm{CS}^{-}$, conditioned stimuli $\left(\mathrm{CS}^{+}\right)$ were associated with an increasingly more negative going slow wave in the delay interval (between $\mathrm{CS}^{+}[t=0 \mathrm{~s}]$ and US onset $[t=2 \mathrm{~s}])$. This was reflected in a sustained negative cluster with strongest polarity at centroparietal electrodes (i.e., parietal slow wave; Fig. 4). Following the onset of the reinforcement (US), in $\mathrm{CS}^{+}$trials the ERP rapidly flipped its polarity while maintaining a highly similar parietal topography, thus giving rise to a sustained positive cluster (i.e., late parietal potential [LPP]; Fig. 4). In line with the established role of anticipatory slow waves and LPPs in arousal and emotion processing (for reviews, see van Boxtel and Böcker, 2004; Schupp et al., 2006; Vazey et al., 2018), this points to increased sustained attention to $\mathrm{CS}^{+}$during the anticipatory delay interval ( $0-2$ s) and an augmented arousal response following US ( $>t=2 \mathrm{~s}$ ). Both the topography and time course of the ERP responses were highly similar across age groups, indicating a maintained arousal response to conditioned stimuli $\left(\mathrm{CS}^{+}\right.$; and US) across the lifespan.

Notably, as the earlier anticipatory potential emerges before onset of the reinforcement, we can rule out that it constitutes an artifact of the somatosensory stimulation. The latter potential in contrast may be influenced by the reinforcement and should only be considered further if it is reliably reinstated in absence of the stimulation (see below).

\section{Reinstatement of parietal ERPs in younger adults and older adults}

ERPs to the arousal manipulation were reinstated in the dichotic listening task in the absence of reinforcements (US). Across YA and OA, both the earlier, anticipatory negative potential as well as the later, positive potential reached significance (negative: $W$ $(77)=861 ; Z=-3.252 ; p=0.001$; positive: $W(77)=2028 ; Z=$ $2.673 ; p=0.008)$. While in YA, only the anticipatory response was reliably reinstated (negative: $W(39)=214 ; Z=-2.456$; $p=0.014$; positive: $W(39)=456 ; Z=0.921 ; p=0.357$; Fig. 5), in OA both reached statistical significance (negative: $W(38)=214$; $Z=-2.270 ; p=0.023$; positive: $W(38)=582 ; Z=3.067 ; p=0.002$; Fig. 5). The age difference in the latter, positive response was marginally significant (YA vs $\mathrm{OA}: U(77)=1335 ; Z=$ $-1.890 ; p=0.059)$. In the absence of somatosensory stimulation and associated artifacts, the reinstatement of the parietal reconditioning ERPs is attributed to the arousal response following the reactivation of the fear memory (van Boxtel and Böcker, 2004; Schupp et al., 2006).

Fear-conditioned posterior desynchronization in younger adults and older adults

Across age groups, fear-conditioned stimuli were also associated with a sustained decrease in low EEG frequencies. Figure $6 a$ 

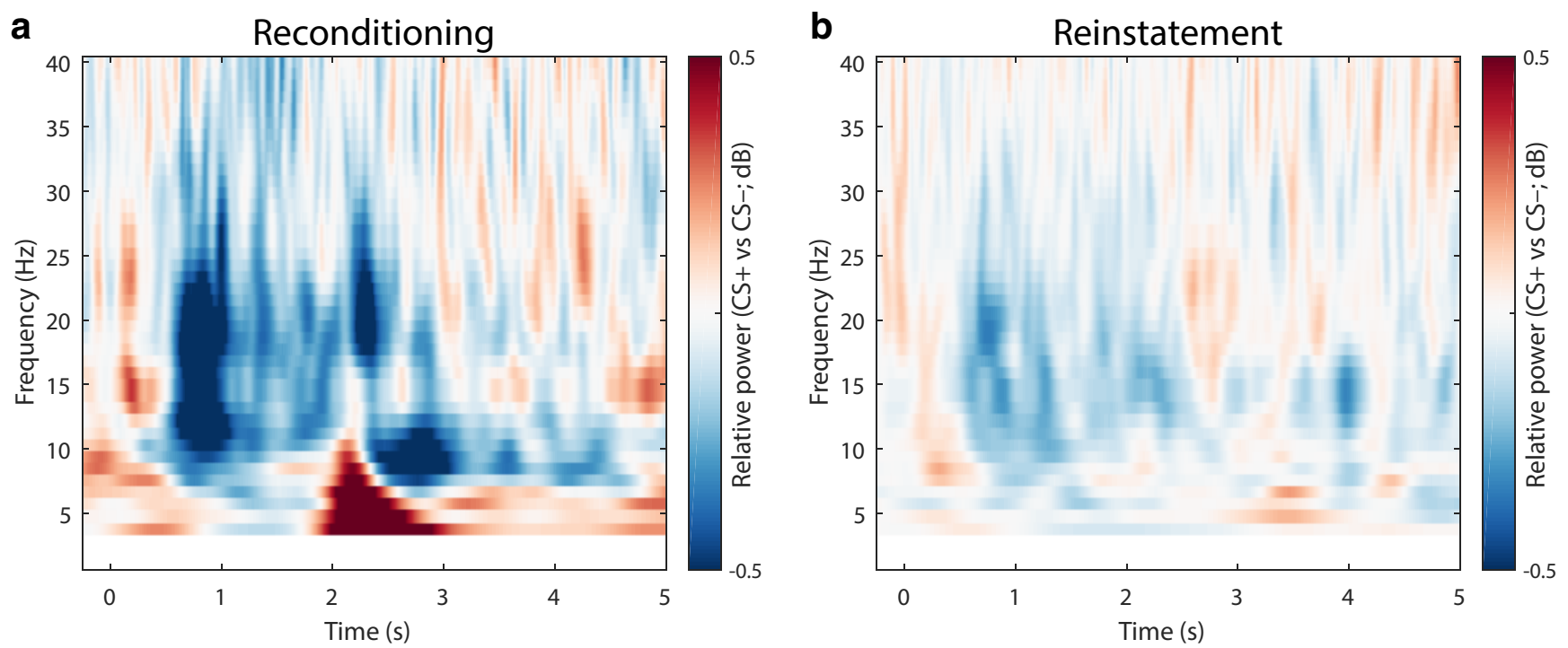

Figure 6. Difference in spectral responses to the presentation of fear-conditioned $\left(\mathrm{CS}^{+}\right)$and neutral control stimuli $\left(\mathrm{CS}^{-}\right)$collapsed over age groups (YA and $\left.\mathrm{OA}\right)$. During reconditioning trials $(\boldsymbol{a}), \mathrm{CS}^{+}$elicit a stronger low-frequency desynchronization compared with $\mathrm{CS}^{-}$. During the dichotic listening task $(\boldsymbol{b})$, the low-frequency desynchronization is reinstated in the absence of somatosensory stimulation. For each frequency, the raw power values are expressed as the relative power ( $\mathrm{dB})$ with respect to the power in a baseline interval $(t=-1.5-0 \mathrm{~s}$, with respect to $C \mathrm{~S}$ onset). After baseline correction, the difference between conditions is calculated $\left(\mathrm{CS}^{+}\right.$vs $\left.\mathrm{CS}^{-}\right)$. For visualization, data are averaged across all electrodes. For associated statistics, see Figure 7.

shows the differences in relative power changes between arousal conditions $\left(\mathrm{CS}^{+}\right.$vs $\left.\mathrm{CS}^{-}\right)$during reconditioning trials (for statistics, see Fig. 7).

Accordingly, cluster statistics across age groups revealed a reliable desynchronization effect. The observed cluster $(t=$ $-0.248-4.956 \mathrm{~s})$ spanned a broad frequency spectrum $(4-40 \mathrm{~Hz})$ but was most pronounced in the $\theta$ to $\beta$ frequency bands $(\sim 5-$ $25 \mathrm{~Hz}$ ) with strongest polarity at parieto-occipital electrodes (compare van Boxtel and Böcker, 2004; YA and OA: $p_{\text {corr }}=$ 0.002). Analyses within YAs and OAs demonstrated reliable desynchronization effects in both age groups with strongest extent at posterior electrodes $\left(\mathrm{YA}: p_{\text {corr }}=0.024\right.$; OA: $p_{\text {corr }}=$ 0.002; Fig. 7a). However, in YA, desynchronization was restricted to the anticipatory delay phase (i.e., before US onset; $t=0.352-1.360 \mathrm{~s} ; 5-40 \mathrm{~Hz}$ ); whereas in $\mathrm{OA}$, a more persistent desynchronization was observed $(t=-0.248-5.144 \mathrm{~s} ; 2-40 \mathrm{~Hz})$. In addition, coinciding with onset of the reinforcement (US), a positive low-frequency cluster emerged across and within age groups (Fig. 7a). However, this positive cluster most likely reflects an artifact of the electric stimulation ( $2 \mathrm{~Hz}$ pulse at $t=2 \mathrm{~s})$ and consequently was not evident in absence of the US (Fig. 7b).

Desynchronization in low frequencies has been associated with NE-associated changes in cortical and behavioral state (McCormick et al., 1991; Harris and Thiele, 2011; Marzo et al., 2014; Safaai et al., 2015; Neves et al., 2018). Moreover, in humans, desynchronization in the $\alpha-\beta$ band was linked to facilitated information processing resulting from a decrease in cortical inhibition (for review, see Klimesch et al., 2007; Jensen and Mazaheri, 2010; Hanslmayr et al., 2012). Accordingly, the pronounced low-frequency desynchronization in response to conditioned stimuli $\left(\mathrm{CS}^{+}\right.$; and US) suggests an anticipatory transition toward a more activated cortical state, including increased cortical excitability and attention deployment.

\section{Reinstatement of posterior desynchronization in younger adults and older adults}

Presentation of fear-conditioned stimuli $\left(\mathrm{CS}^{+}\right)$during the dichotic listening task (i.e., without reinforcements; US) reinstated a pronounced decrease in low frequencies across age groups (YA and OA: $W(77)=900 ; Z=-3.054 ; p=0.002$ ). Similarly, within YA, we observed a reliable reinstatement with a mostly posterior topography (YA: $W(39)=161 ; Z=-3.196 ; p=0.001$ ), whereas OA showed a marginally significant reinstatement with a more widespread extent $(\mathrm{OA}: W(38)=239 ; Z=-1.907 ; p=0.057 ;$ Fig. $7 b)$. As for the pupillary and parietal ERP reinstatement, the reinstatement of the low-frequency desynchronization is considered as arousal response to the reactivated fear memory.

\section{Reinstatement of EEG arousal responses is associated with pupil dilation}

To briefly summarize the modality-specific findings, across groups we observed a negative, anticipatory slow wave and a late, positive parietal potential (ERP; Fig. 4; van Boxtel and Böcker, 2004; Schupp et al., 2006), a low-frequency desynchronization (ERD; Figs. 6, 7; McCormick et al., 1991; Harris and Thiele, 2011; Marzo et al., 2014), as well as pupil dilation (ERPR; Fig. 3; Joshi et al., 2016; Reimer et al., 2016; Breton-Provencher and Sur, 2019; Deitcher et al., 2019; Zerbi et al., 2019). Within the dichotic listening task (i.e., in the absence of reinforcements; US; Fig. 1), we largely witnessed a reliable reinstatement of the arousal response across modalities (i.e., ERP, ERD, ERPR; Figs. 3, 5, and 7). We interpret the reinstatement of the arousal response as reflecting a phasic activation of the LCNE system by the reactivated fear memory. To support this claim, electrophysiological reinstatement marker (ERP, ERD) should be linked to pupil reinstatement, a noninvasive index of LC activity.

Indeed, cluster permutation correlations revealed a reliable association between the reinstatement of the anticipatory, parietal slow wave and pupillary reinstatement $\left(p_{\text {corr }}=0.028\right)$. The cluster reached significance in the time window previously filled by the reinforcement (during (re)conditioning; $t=2.108-2.212 \mathrm{~s}$; Figs. 1,8$)$ and showed its strongest polarity at left lateralized centroparietal electrodes. Spearman correlation coefficients are reported in Table 3 for analyses across 


\section{a Reconditioning}
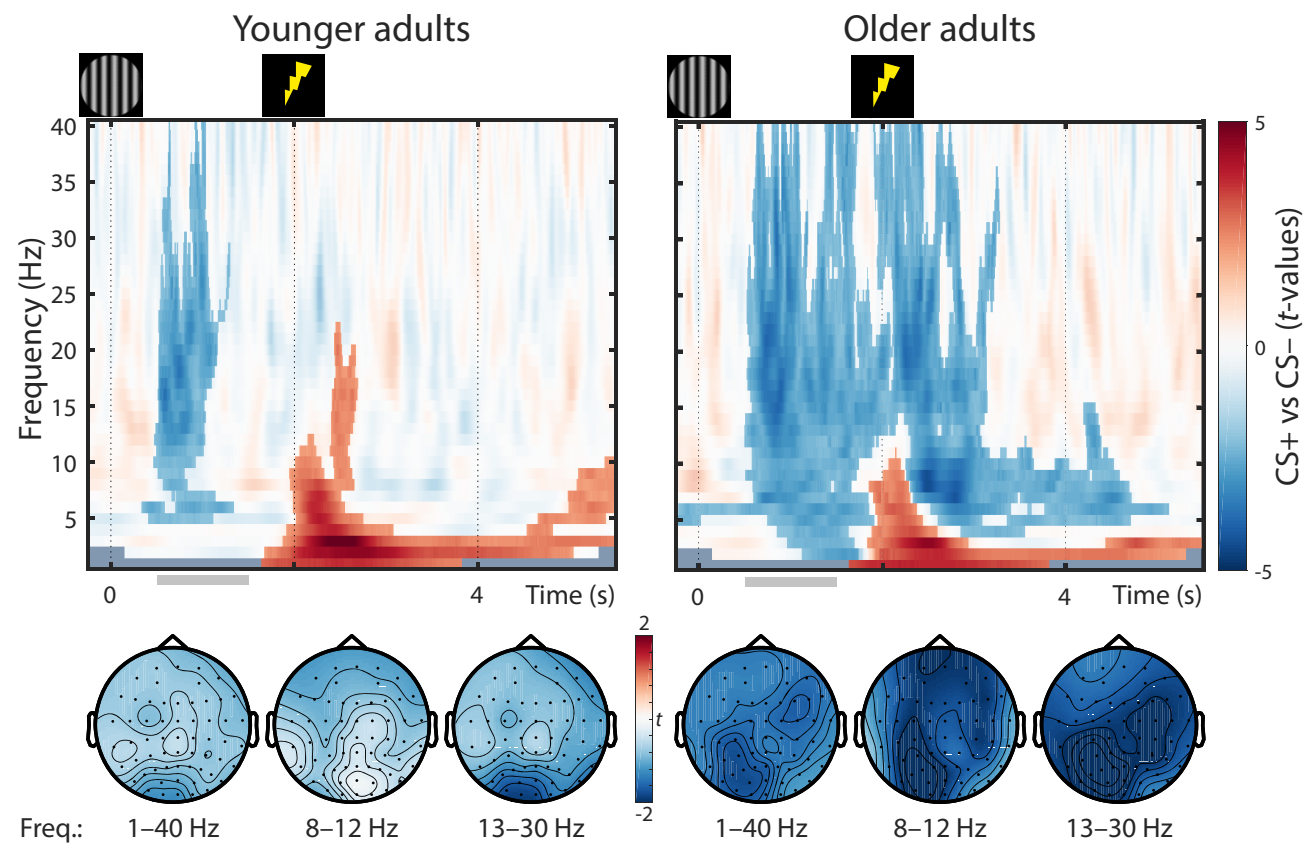

\section{b Reinstatement}
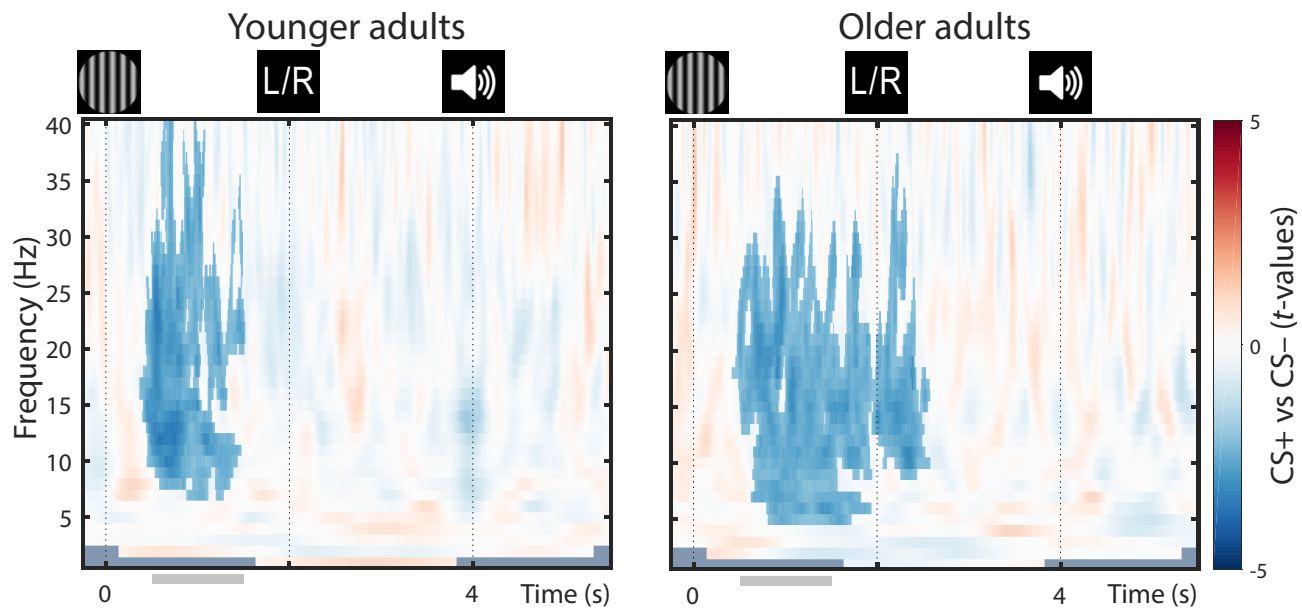

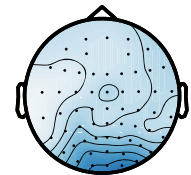

Freq.

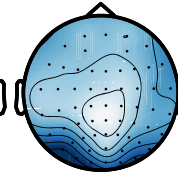

$8-12 \mathrm{~Hz}$

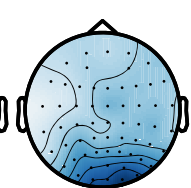

$13-30 \mathrm{~Hz}$
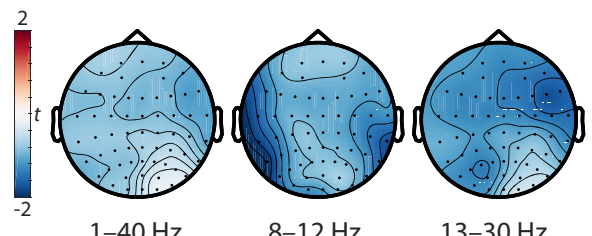

Figure 7. Group statistics for YA (left) and OA (right): time-frequency EEG responses during reconditioning trials on day 2 (a; i.e., Event-Related Desynchronization (ERD)) as well as during dichotic listening trials on day $2\left(\boldsymbol{b}\right.$; i.e., reinstatement of the ERD). Statistics depict the consistency of the $\mathrm{CS}^{+}$versus $\mathrm{CS}^{-}$contrast on the second level. Nonsignificant samples are displayed with $50 \%$ transparency, whereas significant clusters $\left(p_{\text {corr }}<0.05\right)$ are overlaid without transparency. The topography between 0.5 and $1.5 \mathrm{~s}$ relative to $C S$ onset is shown below the time courses (gray horizontal bars). For topographies, data are averaged across all frequencies (left), across the $\alpha$ band (middle), and across the $\beta$ frequency band (right).

and within groups (for this, EEG data were averaged across the cluster). No reliable link was observed between the second, positive ERP cluster (LPP) and pupil dilation $\left(t>2 \mathrm{~s}\right.$; $p_{\text {corr }}>$ $0.1)$. This indicates that the LPP was less reliably linked to our LC-NE activity index, and we thus dropped it from further analyses.
Moreover, a stronger reinstatement of low EEG frequency desynchronization in response to conditioned stimuli $\left(\mathrm{CS}^{+}\right.$vs $\left.\mathrm{CS}^{-}\right)$ was associated with a larger reinstatement of pupil dilation $\left(p_{\text {corr }}=\right.$ 0.022 ). The cluster reached significance in the anticipatory delay phase (i.e., before US onset during (re)conditioning; $t=0.588-1.160 \mathrm{~s}$ ) in the $\alpha-\beta$ frequency band $(9-30 \mathrm{~Hz})$ and was most pronounced at 


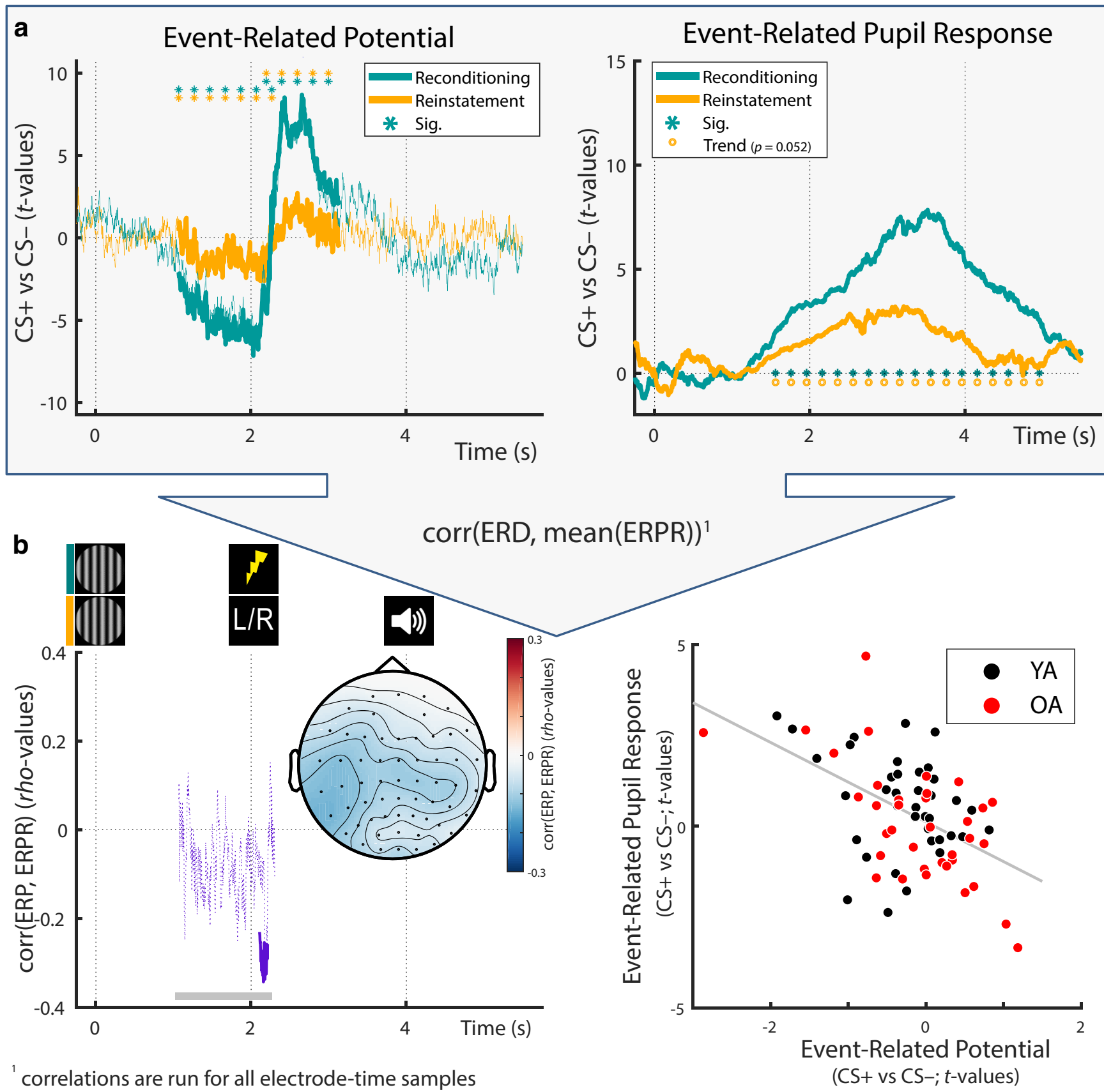

Figure 8. $\quad \boldsymbol{a}$, Across age groups, fear-conditioned Event-Related Potentials (ERP) and Event-Related Pupil Responses (ERPR) to the arousal manipulation learned during re/conditioning (teal lines) were reinstated in the dichotic listening task (orange lines in the left and right, respectively). For ERPR and ERP analyses within age groups, see Figure 3 and Figures 4 and 5 , respectively. $\boldsymbol{b}$, Left, Within electrode and time ranges that demonstrated a reliable arousal effect during reconditioning, ERP and ERPR reinstatement data were correlated. A significant negative association was observed between $t=2.108$ and $t=2.212 \mathrm{~s}$ (solid purple line). The topography between 1 and $2.3 \mathrm{~s}$ relative to CS onset (see gray horizontal bars) is shown below the time course. Right, For visualization purposes only, an additional scatter plot is provided, depicting the same association between ERP and ERPR reinstatement data (here ERP data are averaged over those samples [time, electrodes] that formed the reliable cluster [for cluster extent, see left]). Corr, Correlation; $\mathrm{CS}^{+}$: Conditioned stimulus; $\mathrm{CS}^{-}$: Neutral control stimulus, perceptually matched to the $\mathrm{CS}^{+}$.

parieto-occipital electrodes (Fig. 9). Spearman correlation coefficients (based on EEG data averaged across the cluster) are provided in Table 3.

In sum, in line with our interpretation, cluster correlations indicated that both ERP and ERD responses to the arousal manipulation were linked to pupil dilation, a proxy for LC-NE activity. To further corroborate this conclusion, we repeated our analyses, this time using reconditioning instead of reinstatement pupil and EEG data (compare Fig. 1c). We again observed a reliable, qualitatively similar, association between EEG responses and pupil dilation, suggesting a common dependence on LC-NE activity (ERP: $p=0.026$ and $p=0.044$ [two reliable clusters], $t=1.12-1.218 \mathrm{~s}$ and $t=1.706-1.832 \mathrm{~s}$; ERD: $p=0.03, t=0.648-1.112 \mathrm{~s}$, frequency range $=13-37 \mathrm{~Hz}$ ). Crucially, we additionally repeated these analyses on the single-trial level. That is, we tested whether we could predict a given trial's phasic pupil dilation based on its ERP and ERD data. We obtained findings qualitatively similar to the here reported betweensubject analyses (see Control analyses). 
Table 3. Overview of associations between pupillary and EEG reinstatement components $^{a}$

\begin{tabular}{lllr}
\hline Measure & Group $(N)$ & rho & \multicolumn{1}{c}{$p$} \\
\hline Parietal slow wave & YA + OA (76) & -0.424 & $<0.001$ \\
$\alpha$ - $\beta$ desynchronization & YA + OA (76) & -0.424 & $<0.001$ \\
Parietal slow wave & YA (39) & -0.255 & 0.116 \\
$\alpha-\beta$ desynchronization & YA (39) & -0.401 & 0.011 \\
Parietal slow wave & OA (37) & -0.567 & $<0.001$ \\
$\alpha-\beta$ desynchronization & OA (37) & -0.411 & 0.011 \\
\hline
\end{tabular}

${ }^{a}$ Pupil and EEG reinstatement was assessed in the dichotic listening task (compare Fig. 1c). The first row within age groups reports Spearman's correlations of pupil dilation and the anticipatory time domain EEG cluster, and the second row reports the time-frequency domain EEG cluster.

\section{Multimodal assessment of noradrenergic responsiveness is linked to selective attention in younger adults and older adults}

We integrated over (pupil-associated) EEG and pupil dilation markers to derive a single, latent multimodal measure reflecting LC-NE responsiveness (Fig. 10, top). The proposed model fit the data well $\left(\chi^{2}=6.935, \mathrm{df}=16\right.$; RMSEA $=0.0$; CFI $=1.205$; Brown, 2006). The variances of the latent factors differed reliably from zero in each age group (all $\Delta \chi^{2} \geq 23.845, \Delta \mathrm{df}=1$, all $p<0.001$ ), indicating interindividual differences in $\mathrm{NE}$ responsiveness. Older age was associated with lower NE responsiveness scores $(\rho=-0.301 ; p=0.006$; Fig. $11 a)$.

To evaluate the behavioral relevance of interindividual differences in NE responsiveness in YA and OA, we merged our general attention (see above) and NE responsiveness structural equation modeling in a unified neurocognitive model that demonstrated good fit to the data $\left(\chi^{2}=45.624, \mathrm{df}=85\right.$; $\mathrm{RMSEA}=$ 0.0 ; CFI = 1.625; Fig. 10; Brown, 2006).

Importantly, general attention was positively associated with latent NE responsiveness scores in both YA and OA (YA: $\Delta \chi^{2}=$ $10.323, \Delta \mathrm{df}=1, p=0.001$, standardized estimate $[\mathrm{SE}]=0.75$; OA: $\Delta \chi^{2}=7.262, \Delta \mathrm{df}=1, p=0.007, \mathrm{SE}=0.52$; Fig. $\left.11 b\right)$. The strength of the NE-attention association did not differ reliably between age groups $\left(\Delta \chi^{2}=0.003, \Delta \mathrm{df}=1, p=0.954\right)$. This indicates that, in the face of declining selective attention in aging, a responsive NE system was linked to preserved cognitive abilities (Nyberg et al., 2012).

Notably, qualitatively similar results were obtained when we analyzing composite scores of noradrenergic responsiveness and selective attention (i.e., without relying on a structural equation model). In particular, simple composite scores were derived by averaging across (1) physiological (ERPR, ERP, and ERD) variables for the NE factor and (2) performance in the four cognitive tasks for the attention factor. Crucially, this (less sensitive) analysis approach again indicates an association between NE responsiveness and attention (YA + OA: $\rho=0.436, p<0.001$; YA: $\rho=$ $0.576 ; p<0.001$; OA: $r=0.284 ; p=0.085$; difference between YA and OA's correlation coefficients: $Z=1.535, p=0.125$ ).

\section{Control analyses}

We conducted a set of control analyses to evaluate the effect of moment-to-moment fluctuations in arousal on behavior. The full model (compare Eq. 2; AIC: 17,547; BIC: 17,652, adjusted $r^{2}=$ 0.040 ) better fit the data compared with a model without physiological predictors, as indicated by a likelihood-ratio test $(\mathrm{df}=9)$ $=476.54, p<0.001$ ). However, none of the individual physiological predictors reliably predicted single-trial performance (Table $4)$. We noted a statistical trend for better performance with larger pupil dilation $(p=0.079)$ and stronger low-frequency desynchronization $(p=0.073)$ during arousal trials.
While our analyses lacked the sensitivity to detect arousalbehavior associations on a single trial level, we observed a statistical trend toward better performance in arousal trials $\left(\mathrm{CS}^{+}\right)$with larger pupil dilation and stronger desynchronization. Thus, it appears that the arousal manipulation did affect performance in the dichotic listening task in those instances in which it was associated with larger pupil dilation and stronger desynchronization.

In a second set of control analyses, we evaluated the association between moment-to-moment fluctuations in pupil dilation and arousal-related EEG parameters (ERP, ERD). The full model (AIC: 22,123; BIC: 22,197, adjusted $r^{2}=0.613$ ) better fit the data relative to a model without physiological factors (likelihood-ratio test $(\mathrm{df}=5)=12,173, p<0.001)$. As detailed in Table 5, all physiological predictors were related to phasic pupil dilation. Most importantly, we observed that phasic pupil dilation in a given trial was associated with each trial's low-frequency desynchronization and parietal ERP (Table 5), replicating the between-person results and previous animal work (see Figs. 8, 9; compare McGinley et al., 2015; Stitt et al., 2018).

\section{Discussion}

Animal studies suggest that attention deficits in aging are linked to altered central noradrenergic activity (Arnsten and GoldmanRakic, 1985; Wang et al., 2011). In vivo research in aging humans, however, was long hampered by methodological challenges in the reliable assessment of LC-NE activity (Astafiev et al., 2010). Here we build on recent reports that pupil dilation (Joshi et al., 2016; Reimer et al., 2016; Breton-Provencher and Sur, 2019; Deitcher et al., 2019; Zerbi et al., 2019) and certain event-related EEG components (Harris and Thiele, 2011; Marzo et al., 2014; Neves et al., 2018; Vazey et al., 2018) are valid, noninvasive proxies for noradrenergic activity. In particular, we made use of LC-NE's well-established role in fear processing (Szabadi, 2012; Uematsu et al., 2017; Likhtik and Johansen, 2019) to experimentally test the responsiveness of the central noradrenergic system while recording pupil dilation and the EEG. In addition, we applied a multimodal assessment to probe general attention performance in samples of healthy YA and OA. Our findings demonstrate impaired attention in aging across multiple tasks. Moreover, older age was associated with a reduced NE responsiveness as indexed by pupil dilation and EEG. Crucially, within both YA and OA, individual differences in attention were positively related to the responsiveness of the noradrenergic system.

On the behavioral level, both YA and OA demonstrated successful auditory selective attention in a dichotic listening task (Hugdahl et al., 2009). That is, participants in both age groups were able to adapt their attentional focus according to changing demands. However, in line with earlier reports (Passow et al., 2012, 2014; Dahl et al., 2019a), OA showed impaired attention performance in the dichotic listening task and, beyond that, across a variety of alternative attention tasks (Kennedy and Mather, 2019).

On the physiological level, we observed a multimodal response to the arousal manipulation during fear (re)conditioning in YA and OA. In particular, compared with perceptually matched control stimuli $\left(\mathrm{CS}^{-}\right)$, conditioned stimuli $\left(\mathrm{CS}^{+}\right)$elicited a sustained dilation of the pupil, as previously reported (e.g., Lee et al., 2018). In line with recent animal work linking LC activity to pupil dilation, we thus conclude that our manipulation successfully activated the LC-NE system in YA and OA (Rasmussen and Jacobs, 1986; Szabadi, 2012; Uematsu et al., 2017; Deitcher et al., 2019). However, using noninvasive 
a Event-Related Desynchronization

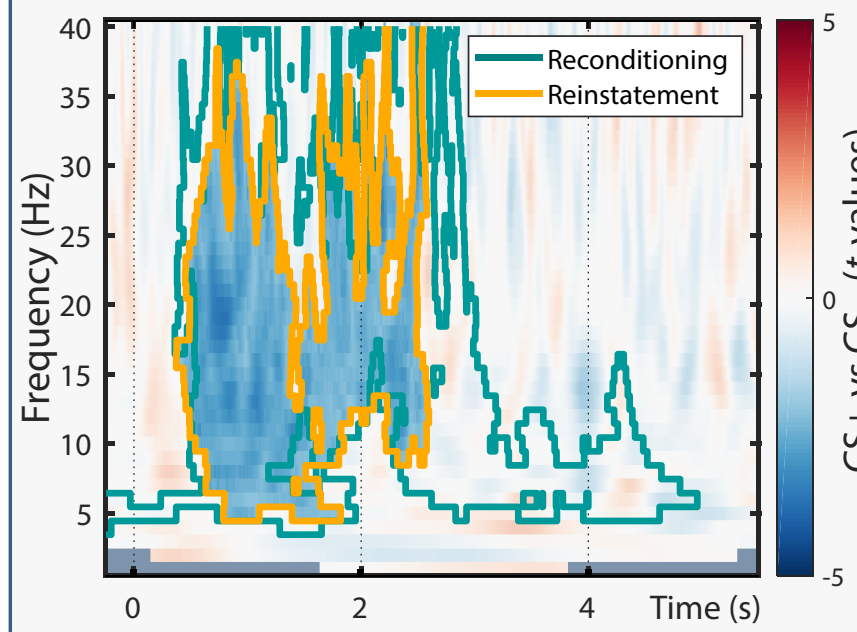

Event-Related Pupil Response
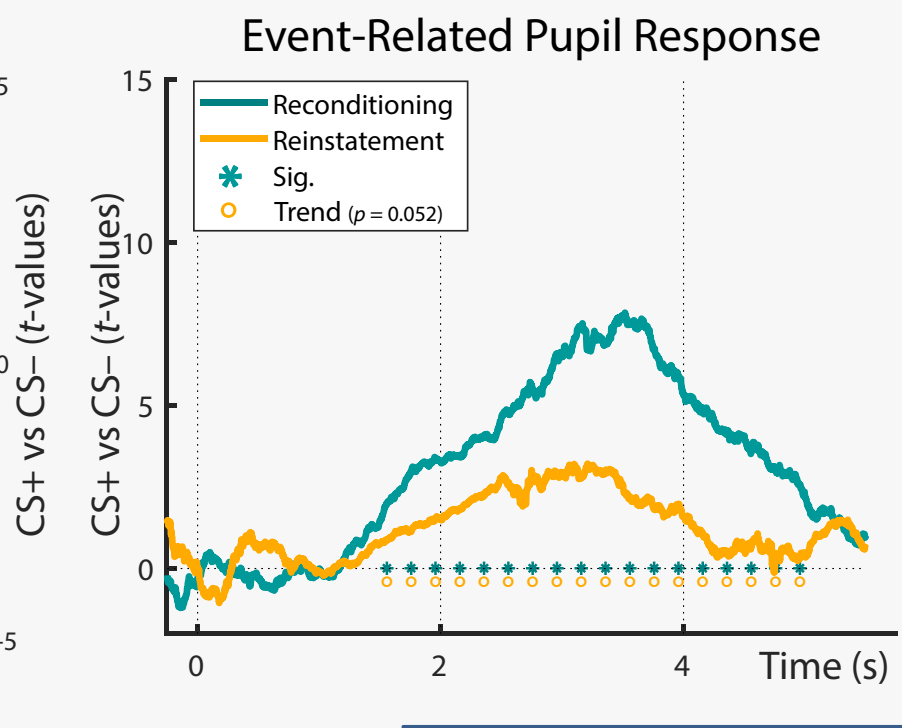

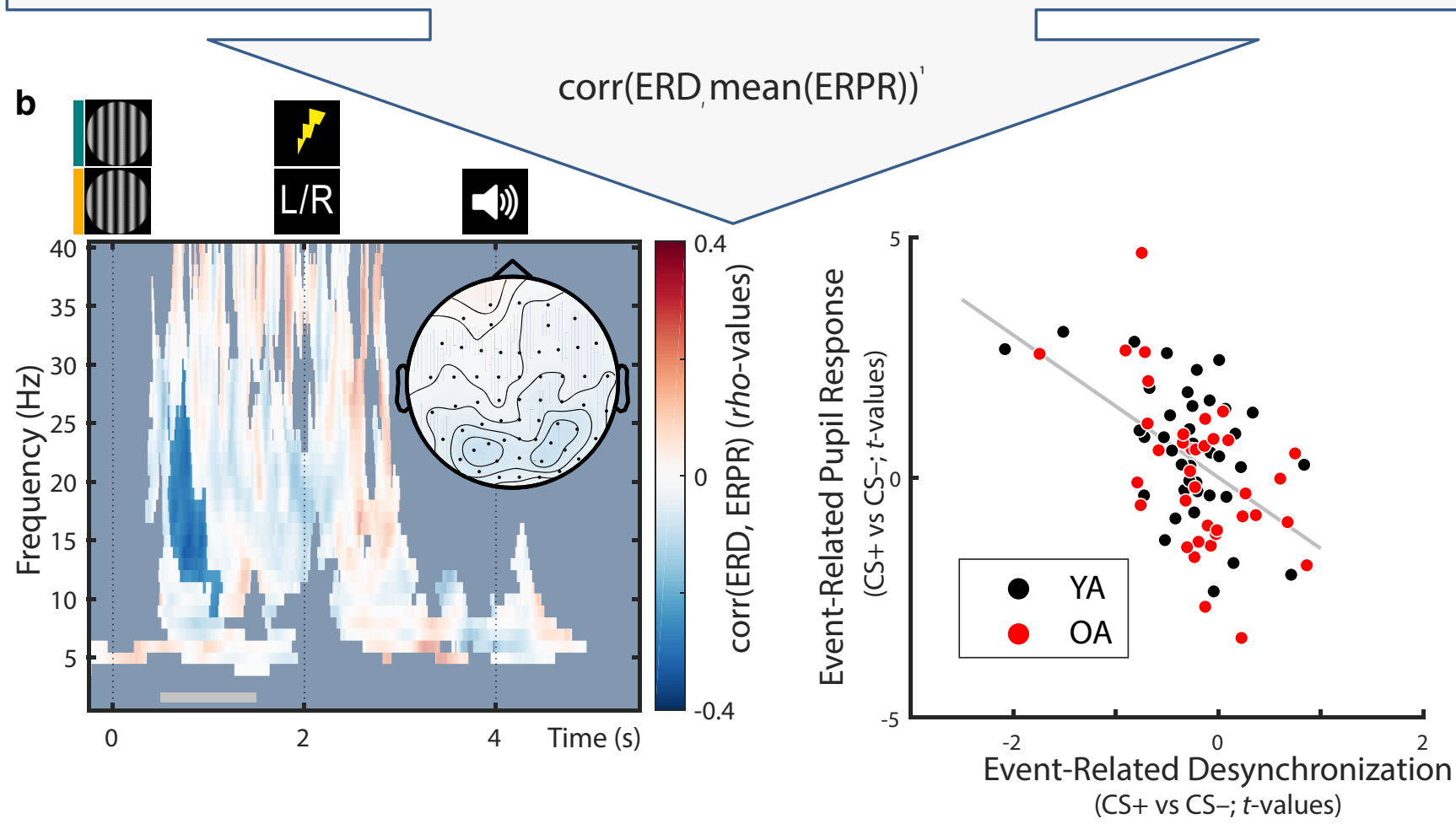

Figure 9. a, Across age groups, fear-conditioned Event-Related Desynchronization (ERD) and Event-Related Pupil Responses (ERPR) to the arousal manipulation learned during re/conditioning (teal (out)lines) were reinstated in the dichotic listening task (orange (out)lines in the left and right, respectively). For ERPR and ERD analyses within age groups, see Figures 3 and 7 , respectively. $\boldsymbol{b}$, Left, Within electrode, time and frequency ranges that demonstrated a reliable arousal effect during reconditioning, ERD and ERPR reinstatement data were correlated. A significant negative association was observed between $t=0.558$ and $t=1.160 \mathrm{~s}$ (nontransparent cluster). The topography between 0.5 and $1.5 \mathrm{~s}$ relative to $\mathrm{CS}$ onset (see gray horizontal bar) is shown below the time course. Right, For visualization purposes only, an additional scatter plot is provided, depicting the same association between ERD and ERPR reinstatement data (here ERD data are averaged over those samples [time, electrodes, frequencies] that formed the reliable cluster [for cluster extent, see left]). Corr, Correlation; CS+: Conditioned stimulus; CS-: Neutral control stimulus, perceptually matched to the $\mathrm{CS}+$. Here higher values on the ERD axis indicate more synchronization, whereas lower values indicate more desynchronization.

measures, we cannot rule out that other arousal-related neuromodulatory systems also influenced pupil diameter (e.g., cholinergic activation; Reimer et al., 2016).

$\mathrm{CS}^{+}$further gave rise to two sustained centroparietal eventrelated EEG components: (1) an anticipatory slow wave; and (2) a late parietal potential that occurred before and after onset of the reinforcement (US), respectively. A comparable slow wave (stimulus-preceding negativity [SPN]) has been observed in response to cues (S1) that prepared participants for the occurrence of following arousing or behaviorally relevant stimuli (S2; compare Breska and Deouell, 2017; for review, see van Boxtel and Böcker, 2004). Concerning its functional relevance, the SPN has been suggested as marker of anticipatory processes that adjust the excitability of cortical networks to facilitate subsequent processing (of S2; Birbaumer et al., 1990). Similarly, Brunia (1993) proposed the SPN as index of regionally targeted changes in cortical excitability that are produced via corticothalamic interactions. Interestingly, various peripheral correlates of noradrenergic activation (e.g., skin conductance, heart rate; compare Szabadi, 2013) have been observed concomitant with the SPN 


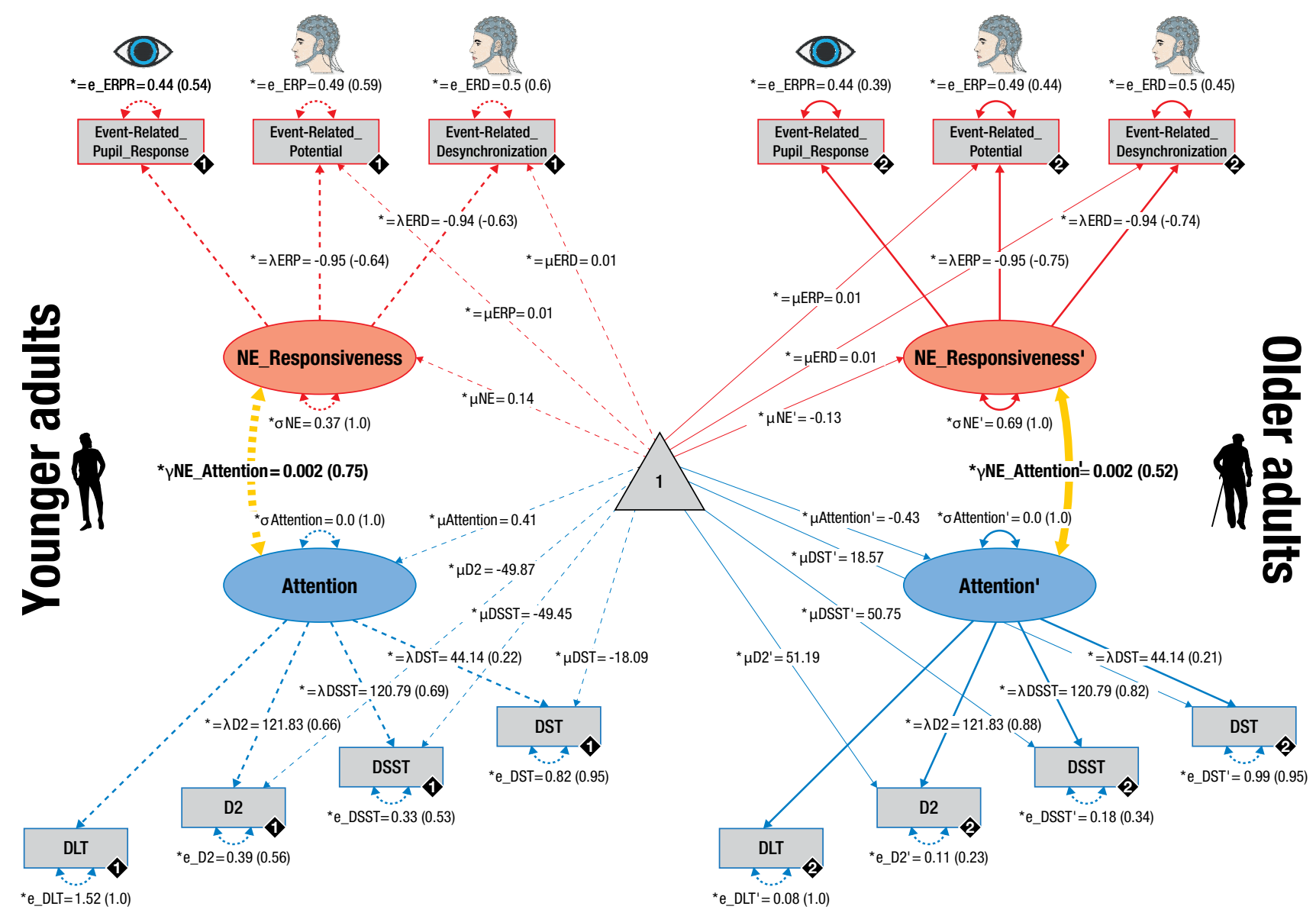

Figure 10. Pictorial rendition of the structural equation model that probes associations (yellow lines) between noradrenergic responsiveness (NE_Responsiveness; red) and attention performance (blue) in YA and OA on a latent level. Rectangles and ellipses represent manifest (observed) and latent variables, respectively. The triangle represents the constant. Cognitive manifest variables represent attention performance assessed in a Dichotic Listening Task (DLT; compare Figs. 1, 2), D2 task of attention (compare Table 2), DSST, and DST. Physiologic manifest variables represent the reinstatement of fear-conditioned ERPRs (compare Fig. 3), ERPs (compare Fig. 5), and ERDs (compare Fig. 7). Black diamonds on manifest variables represent the age group $(\mathrm{YA}=1$, broken lines; $\mathrm{OA}=2$, solid lines). (Co)variances $(\gamma, \sigma)$ and loadings $(\lambda)$ in parentheses indicate standardized estimates. Loadings that are freely estimated $(*)$ but constrained to be equal across age groups (=) are indicated by both asterisk and equal signs $\left.{ }^{*}=\right)$. The cognitive submodel demonstrated metric factorial invariance (invariant manifest means and errors across age groups), whereas the physiological submodel showed strict factorial invariance (manifest means and errors are constrained across groups).

(van Boxtel and Böcker, 2004; Poli et al., 2007). Together, a larger SPN $\left(\mathrm{CS}^{+}\right.$vs $\left.\mathrm{CS}^{-}\right)$points to a heightened anticipatory attention deployment in arousing situations in YA and OA. After presentation of the reinforcement (US), $\mathrm{CS}^{+}$were associated with a second sustained parietal event-related component (LPP). Previous studies have observed the LPP during fear conditioning (Bacigalupo and Luck, 2018) and suggested it as index of facilitated attention allocation to arousing stimuli (for review, see Schupp et al., 2006). Furthermore, the LPP has been linked with peripheral markers of noradrenergic activity (e.g., skin conductance response; compare Szabadi, 2013) and subjective arousal ratings. In line with previous work, we interpret the LPP as reflecting elevated selective attention during arousing conditions in YA and OA.

In addition to ERP, fear conditioned stimuli $\left(\mathrm{CS}^{+}\right)$produced pronounced changes in rhythmic neural activity within YA and $\mathrm{OA}$. We observed an anticipatory, long-lasting desynchronization in low EEG frequencies (ERD) with strongest magnitude at parieto-occipital electrodes (van Boxtel and Böcker, 2004). Increased activity in neuromodulatory nuclei like the LC causes global cortical desynchronization (i.e., cortical state changes; McCormick et al., 1991; Marzo et al., 2014; Neves et al., 2018). Of note, the neural patterns associated with cortical state changes
Table 4. Fixed-effect coefficients for single-trial logistic regression analyses with accuracy as outcome ${ }^{a}$

\begin{tabular}{lrlrll}
\hline Fixed effects & Estimate & SE & \multicolumn{1}{l}{$t$} & $\mathrm{df}$ & $p$ \\
\hline Intercept* $^{*}$ & 0.145 & 0.062 & 2.335 & 12,979 & 0.02 \\
CS $^{+}$ & 0.039 & 0.036 & 1.092 & 12,979 & 0.275 \\
Attentional_condition: right* $^{*}$ & 0.377 & 0.036 & 10.487 & 12,979 & 0 \\
Old_age* $^{*}$ & -0.323 & 0.081 & -3.985 & 12,979 & 0 \\
ERP & 0.023 & 0.12 & 0.196 & 12,979 & 0.845 \\
ERP_baseline & -0.042 & 0.085 & -0.494 & 12,979 & 0.621 \\
Pupil & -0.029 & 0.038 & -0.765 & 12,979 & 0.444 \\
Pupil_baseline & 0.004 & 0.032 & 0.121 & 12,979 & 0.904 \\
ERD & 0.136 & 0.146 & 0.934 & 12,979 & 0.35 \\
ERD_baseline & -0.024 & 0.087 & -0.281 & 12,979 & 0.779 \\
CS $^{+}$:ERP & 0.052 & 0.163 & 0.319 & 12,979 & 0.749 \\
CS $^{+}$:Pupil* & 0.071 & 0.04 & 1.759 & 12,979 & 0.079 \\
CS $^{+}$:ERD* & -0.355 & 0.198 & -1.791 & 12,979 & 0.073 \\
\hline CS $^{+}:$CNdtring $^{*}$ & & & &
\end{tabular}

${ }^{a} \mathrm{CS}^{+}$: Conditioned stimulus; ERP: Event-related potential (parietal slow wave); ERD: Event-related desynchronization; SE: Standard error; Model formula is expressed in Equation 2. Please note that $\mathrm{CS}^{+}$: * indicates the interaction term (:) between $\mathrm{CS}^{+}$and physiological variables.

*Statistically reliable predictors and statistical trends.

and selective attention are highly similar (Harris and Thiele, 2011; Thiele and Bellgrove, 2018). In particular, the global, LCNE-mediated cortical desynchronization may achieve the spatial precision necessary to selectively process attended stimuli in 
a

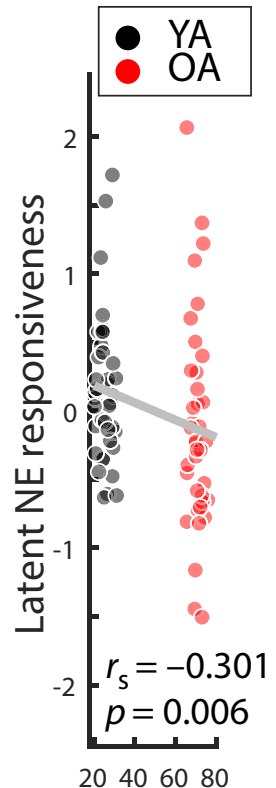

Age (years) b

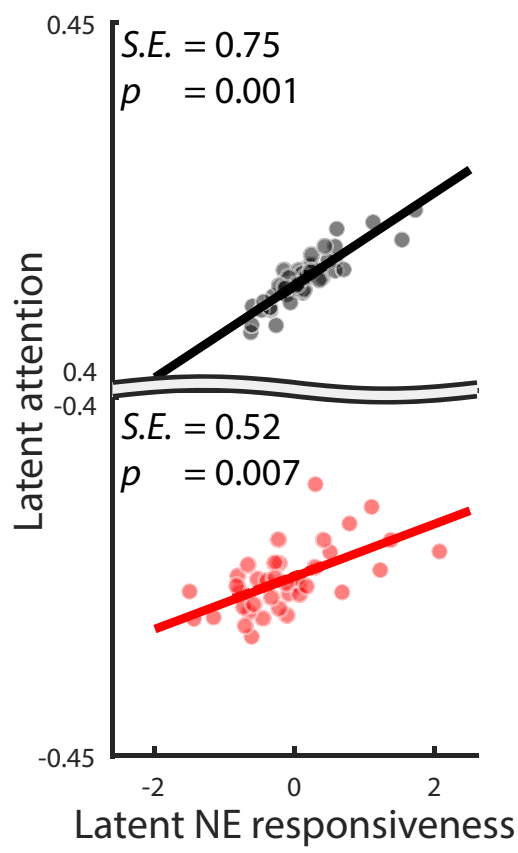

Figure 11. Associations between $(\boldsymbol{a})$ age and latent NE responsiveness, evaluated using Spearman's correlation, as well as $(\boldsymbol{b})$ latent NE responsiveness and latent attention in YA (black) and OA (red). Note the broken y axis in $\boldsymbol{b}$. S.E. = standardized estimate.

interaction with glutamate (Harris and Thiele, 2011; Mather et al., 2016). A wide range of human EEG studies established desynchronization in the $\alpha$ - $\beta$ range as a marker of decreased cortical inhibition that allows for facilitated information processing (Klimesch et al., 2007; Jensen and Mazaheri, 2010; Hanslmayr et al., 2012). Using a similar fear conditioning procedure in rats, Headley and Weinberger (2013) demonstrated that the $\mathrm{CS}^{+}$induced decrease in low frequencies is accompanied by a strong increase in high-frequency multiunit activity $(\gamma ; 40-$ $120 \mathrm{~Hz}$ ), indicating facilitated feedforward processing (Fries, $2005,2015)$. In line with earlier work, the pronounced low-frequency desynchronization in response to CS (and US) suggests a transition toward a more activated cortical state, including increased cortical excitability and attention deployment.

Most of the arousal responses observed during (re)conditioning (i.e., ERPR, ERP, ERD) persisted in both age groups and were reinstated during the dichotic listening task in the absence of reinforcements (US). As a notable exception, in OA, pupil reinstatement did not reach significance on a group level, potentially reflecting age-related difficulties in triggering and maintaining self-initiated processing (Lindenberger and Mayr, 2014). That is, during re/conditioning, repeated external reminders (i.e., US) may have supported OA and thus obscured age differences in pupil responses. In contrast, the lack of this external support during the dichotic listening task may have specifically affected $\mathrm{OA}$ and revealed underlying age-related differences in fear conditionability (LaBar et al., 2004) and the central noradrenergic system (Betts et al., 2017; Dahl et al., 2019b; Liu et al., 2019). In line with this notion, OA demonstrated a reliable modulation of pupil dilation during phases of high external support (i.e., encoding of series of visually presented digits) but no significant modulation during phases requiring more self-initiated processing (i.e., cued recall; see Van Gerven et al., 2004).

Table 5. Fixed-effect coefficients for single-trial logistic regression analyses with phasic pupil dilation as outcome ${ }^{a}$

\begin{tabular}{lrlrlr}
\hline Fixed effects & Estimate & SE & \multicolumn{1}{l}{$t$} & df & \multicolumn{1}{c}{$p$} \\
\hline Intercept* $^{*}$ & -0.051 & 0.018 & -2.884 & 12,984 & 0.004 \\
CS $^{+*}$ & 0.038 & 0.01 & 3.832 & 12,984 & $<0.001$ \\
Old_age & -0.003 & 0.024 & -0.116 & 12,984 & 0.908 \\
ERP* $^{*}$ & -0.104 & 0.023 & -4.522 & 12,984 & $<0.001$ \\
ERP_baseline* & -0.052 & 0.023 & -2.207 & 12,984 & 0.027 \\
Pupil_baseline* & 0.766 & 0.006 & 138.185 & 12,984 & $<0.001$ \\
ERD* $^{*}$ & -0.221 & 0.028 & -7.846 & 12,984 & $<0.001$ \\
ERD_baseline* $^{*}$ & -0.082 & 0.024 & -3.449 & 12,984 & 0.001 \\
\hline
\end{tabular}

${ }^{a} \mathrm{CS}^{+}$: Conditioned stimulus; ERP: Event-related potential (parietal slow wave); ERD: Event-related desynchronization; SE: Standard error; Model formula is expressed in Equation 3. *Statistically reliable predictors.

Crucially, however, in both age groups, individual differences in pupil reinstatement were linked to EEG correlates of the arousal response (i.e., SPN-ERP, ERD), suggesting a common underlying factor. The association between pupil dilation (i.e., our index of LC activity) and EEG responses is in line with optogenetic and pharmacological animal studies (Berridge and Waterhouse, 2003; Vazey et al., 2018). In particular, Vazey et al. (2018) demonstrated that LC photoactivation produced a positive cortical ERP $\sim 140-400 \mathrm{~ms}$ after LC stimulation in the absence of sensory input (compare Nieuwenhuis et al., 2005). Both the parietal topography as well the time course of the observed pupil-associated ERP cluster overlap with such an LC-induced parietal positivity (i.e., $108-214 \mathrm{~ms}$ after $t=2$ [i.e., the onset of the reinforcement during (re)conditioning]). Further, pharmacological animal studies causally implicate LC activity in the modulation of cortical and behavioral states (for review, see Berridge and Waterhouse, 2003). This effect is presumably mediated via NE's action in the thalamus and an activation of the basal forebrain (Buzsáki et al., 1988, 1991; McCormick, 1989; McCormick et al., 1991). Behaviorally significant environmental stimuli elicit a reflexive (re)orienting of attention (orienting response) that is tightly linked to LC activity (Bouret and Sara, 2005; Sara and Bouret, 2012). Remarkably, the orienting response is always accompanied by EEG desynchronization and pupil dilation (Sara and Bouret, 2012), supporting a common dependency on LC activity.

We thus integrated over (pupil-associated) EEG and pupil dilation markers to derive a single, multimodal measure reflecting LC-NE responsiveness. We observed a lower NE responsiveness with older age, which complements previous reports of structural age differences in the LC (Betts et al., 2017; Dahl et al., 2019b; Liu et al., 2019) and age differences in LC functional connectivity (Lee et al., 2018). Crucially, within both YA and OA, a higher noradrenergic responsiveness was associated with better selective attention performance (compare Arnsten and Goldman-Rakic, 1985). That is, in the face of declining selective attention in aging, a responsive NE system was linked to preserved cognitive abilities (Nyberg et al., 2012).

Notably, however, as our study reports correlational data, we cannot exclude that better attentional abilities may have facilitated a preferential processing of CS, which in turn may have led to increased noradrenergic drive. In addition, while previous research demonstrated a link between LC activity and pupil dilation (LC $\rightarrow$ pupil), increases in dilation do not necessarily imply that (only) changes in LC-NE activity have occurred (Joshi and Gold, 2020). In this study, we applied an experimental manipulation, fear conditioning, which reliably drives LC-NE activity, as 
indicated by markers of neuronal activity in animals, for example: LC spiking activity (Rasmussen and Jacobs, 1986); $\mathrm{Ca}^{2+}$ responses in LC axons (Deitcher et al., 2019); and c-Fos (Uematsu et al., 2017; compare Szabadi, 2012). Combining a manipulation that elicits LC activation (fear conditioning) with a noninvasive marker sensitive to LC activation (pupil dilation), we believe that our findings are at least partly attributable to the effects of NE. This argument is supported by our finding that pupil dilation was associated with electrophysiological indices that have also been linked to NE activity in invasive animal studies: that is, the P300 ERP (Nieuwenhuis et al., 2005; Vazey and Aston-Jones, 2014); and low-frequency desynchronization (McCormick et al., 1991; Marzo et al., 2014; Neves et al., 2018). Targeting the noradrenergic system from multiple angles, we hope to narrow down our conclusions. While our data suggest an involvement of the noradrenergic system in this study, we do not rule out the involvement of other neuromodulatory systems in fear conditioning and attention (Thiele and Bellgrove, 2018; Likhtik and Johansen, 2019).

In conclusion, we used noninvasive in vivo markers of noradrenergic activity (Joshi et al., 2016; Reimer et al., 2016; Neves et al., 2018; Vazey et al., 2018) to uncover age differences in NE responsiveness. Importantly, structural equation modeling revealed reliable positive associations between $\mathrm{NE}$ responsiveness and attention in both YA and OA. Our findings link animal and human studies on the neural underpinning of selective attention in aging and underscore the importance of the LC-NE system in late-life cognition (Wilson et al., 2013; Mather and Harley, 2016).

\section{References}

Arnsten AF, Goldman-Rakic PS (1985) Alpha 2-adrenergic mechanisms in prefrontal cortex associated with cognitive decline in aged nonhuman primates. Science 230:1273-1276.

Astafiev SV, Snyder AZ, Shulman GL, Corbetta M (2010) Comment on "Modafinil shifts human locus coeruleus to low-tonic, high-phasic activity during functional MRI" and "Homeostatic sleep pressure and responses to sustained attention in the suprachiasmatic area." Science 328:309.

Aston-Jones G, Cohen JD (2005) An integrative theory of locus coeruleusnorepinephrine function: adaptive gain and optimal performance. Annu Rev Neurosci 28:403-450.

Bacigalupo F, Luck SJ (2018) Event-related potential components as measures of aversive conditioning in humans. Psychophysiology 55:e13015.

Bäckman L, Nyberg L, Lindenberger U, Li SC, Farde L (2006) The correlative triad among aging, dopamine, and cognition: current status and future prospects. Neurosci Biobehav Rev 30:791-807.

Bangasser DA, Wiersielis KR, Khantsis S (2016) Sex differences in the locus coeruleus-norepinephrine system and its regulation by stress. Brain Res 1641:177-188.

Berridge CW, Waterhouse BD (2003) The locus coeruleus-noradrenergic system: modulation of behavioral state and state-dependent cognitive processes. Brain Res Brain Res Rev 42:33-84.

Betts MJ, Kirilina E, Otaduy MC, Ivanov D, Acosta-Cabronero J, Callaghan MF, Lambert C, Cardenas-Blanco A, Pine K, Passamonti L, Loane C, Keuken MC, Trujillo P, Lüsebrink F, Mattern H, Liu KY, Priovoulos N, Fliessbach K, Dahl MJ, Maaß A, et al. (2019) Locus coeruleus imaging as a biomarker for noradrenergic dysfunction in neurodegenerative diseases. Brain 142:2558-2571.

Betts MJ, Cardenas-Blanco A, Kanowski M, Jessen F, Düzel E (2017) In vivo MRI assessment of the human locus coeruleus along its rostrocaudal extent in young and older adults. Neuroimage 163:150-159.

Birbaumer N, Elbert T, Canavan AG, Rockstroh B (1990) Slow potentials of the cerebral cortex and behavior. Physiol Rev 70:1-41.

Bouret S, Sara SJ (2005) Network reset: a simplified overarching theory of locus coeruleus noradrenaline function. Trends Neurosci 28:574-582.
Breska A, Deouell LY (2017) Neural mechanisms of rhythm-based temporal prediction: delta phase-locking reflects temporal predictability but not rhythmic entrainment. PLoS Biol 15:e2001665.

Breton-Provencher V, Sur M (2019) Active control of arousal by a locus coeruleus GABAergic circuit. Nat Neurosci 22:218-228.

Brickenkamp R, Zillmer E (1998) The d2 test of attention. Seattle: Hogrefe and Huber.

Brown TA (2006) Confirmatory factor analysis for applied research. New York: Guilford.

Brunia CHM (1993) Waiting in readiness: gating in attention and motor preparation. Psychophysiology 30:327-339.

Buzsáki G, Bickford RG, Ponomareff G, Thal LJ, Mandel R, Gage FH (1988) Nucleus basalis and thalamic control of neocortical activity in the freely moving rat. J Neurosci 8:4007-4026.

Buzsáki G, Kennedy B, Solt VB, Ziegler M (1991) Noradrenergic control of thalamic oscillation: the role of $\alpha-2$ receptors. Eur J Neurosci 3:222229.

Carter ME, Yizhar O, Chikahisa S, Nguyen H, Adamantidis A, Nishino S, Deisseroth K, De Lecea L (2010) Tuning arousal with optogenetic modulation of locus coeruleus neurons. Nat Neurosci 13:1526-1535.

Chamberlin S, Colbert AP, Larsen A (2011) Skin conductance at 24 source (Yuan) acupoints in 8637 patients: influence of age, gender and time of day. J Acupunct Meridian Stud 4:14-23.

Corbetta M, Patel G, Shulman GL (2008) The reorienting system of the human brain: from environment to theory of mind. Neuron 58:306-324.

Costa VD, Rudebeck PH (2016) More than meets the eye: the relationship between pupil size and locus coeruleus activity. Neuron 89:8-10.

Curran PJ, Obeidat K, Losardo D (2010) Twelve frequently asked questions about growth curve modeling. J Cogn Dev 11:121-136.

Dahl MJ, Ilg L, Li SC, Passow S, Werkle-Bergner M (2019a) Diminished prestimulus alpha-lateralization suggests compromised self-initiated attentional control of auditory processing in old age. Neuroimage 197:414424.

Dahl MJ, Mather M, Düzel S, Bodammer NC, Lindenberger U, Kühn S, Werkle-Bergner M (2019b) Rostral locus coeruleus integrity is associated with better memory performance in older adults. Nat Hum Behav 3:1203-1214.

de Gee JW, Knapen T, Donner TH (2014) Decision-related pupil dilation reflects upcoming choice and individual bias. Proc Natl Acad Sci USA 111:E618-E625.

Deitcher Y, Leibner Y, Kutzkel S, Zylbermann N, London M (2019) Nonlinear relationship between multimodal adrenergic responses and local dendritic activity in primary sensory cortices. Avaiable at https:// www.biorxiv.org/content/10.1101/814657v2.

Delorme A, Makeig S (2004) EEGLAB: an open source toolbox for analysis of single-trial EEG dynamics including independent component analysis. J Neurosci Methods 134:9-21.

Desimone R, Duncan JS (1995) Neural mechanisms of selective visual attention. Annu Rev Neurosci 18:193-222.

Dimigen O, Sommer W, Hohlfeld A, Jacobs AM, Kliegl R (2011) Coregistration of eye movements and EEG in natural reading: analyses and review. J Exp Psychol Gen 140:552-572.

Eid M, Gollwitzer M, Schmitt M (2015) Statistik und Forschungsmethoden: Lehrbuch. Weinheim: Beltz.

Folstein MF, Folstein SE, McHugh PR (1975) Mini-Mental State": a practical method for grading the cognitive state of patients for the clinician. J Psychiatr Res 12:189-198.

Fries P (2005) A mechanism for cognitive dynamics: neuronal communication through neuronal coherence. Trends Cogn Sci (Regul Ed) 9:474-480.

Fries P (2015) Rhythms for cognition: communication through coherence. Neuron 88:220-235.

Gagl B, Hawelka S, Hutzler F (2011) Systematic influence of gaze position on pupil size measurement: analysis and correction. Behav Res Methods 43:1171-1181.

Gelbard-Sagiv H, Magidov E, Sharon H, Hendler T, Nir Y (2018) Noradrenaline modulates visual perception and late visually evoked activity. Curr Biol 28:2239-2249.e6.

Gruene TM, Flick K, Stefano A, Shea SD, Shansky RM (2015) Sexually divergent expression of active and passive conditioned fear responses in rats. Elife 4:e11352.

Hämmerer D, Callaghan MF, Hopkins A, Kosciessa J, Betts M, CardenasBlanco A, Kanowski M, Weiskopf N, Dayan P, Dolan RJ, Düzel E (2018) 
Locus coeruleus integrity in old age is selectively related to memories linked with salient negative events. Proc Natl Acad Sci USA 115:22282233.

Hanslmayr S, Staudigl T, Fellner MC (2012) Oscillatory power decreases and long-term memory: the information via desynchronization hypothesis. Front Hum Neurosci 6:74.

Harris KD, Thiele A (2011) Cortical state and attention. Nat Rev Neurosci 12:509-523.

Headley DB, Weinberger NM (2013) Fear conditioning enhances gamma oscillations and their entrainment of neurons representing the conditioned stimulus. J Neurosci 33:5705-5717.

Herrmann CS, Grigutsch M, Busch NA (2005) EEG oscillations and wavelet analysis. In: Event-related potentials: a methods handbook (Handy TC, ed), pp 229-259. Cambridge: Massachusetts Institute of Technology.

Hong L, Walz JM, Sajda P (2014) Your eyes give you away: prestimulus changes in pupil diameter correlate with poststimulus task-related EEG dynamics. PLoS One 9:e91321.

Hugdahl K, Westerhausen R, Alho K, Medvedev S, Laine M, Hämäläinen H (2009) Attention and cognitive control: unfolding the dichotic listening story. Scand J Psychol 50:11-22.

Jensen O, Mazaheri A (2010) Shaping functional architecture by oscillatory alpha activity: gating by inhibition. Front Hum Neurosci 4:186.

Joshi S, Gold JI (2020) Pupil size as a window on neural substrates of cognition. Advance online publication. Retrieved. April 21, 2020. DOI: 10.1016/j.tics.2020.03.005.

Joshi S, Li Y, Kalwani RM, Gold JI (2016) Relationships between pupil diameter and neuronal activity in the locus coeruleus, colliculi, and cingulate cortex. Neuron 89:221-234.

Jung TP, Makeig S, Humphries C, Lee TW, McKeown MJ, Iragui V, Sejnowski TJ (2000) Removing electroencephalographic artifacts by blind source separation. Psychophysiology 37:163-178.

Kennedy BL, Mather M (2019) Neural mechanisms underlying age-related changes in attentional selectivity. In: The aging brain: functional adaptation across adulthood (Samanez-Larkin GR, ed), pp 45-72. Washington, DC: American Psychological Association.

Kievit RA, Brandmaier AM, Ziegler G, van Harmelen AL, de Mooij SM, Moutoussis M, Goodyer IM, Bullmore E, Jones PB, Fonagy P, Lindenberger U, Dolan RJ, NSPN Consortium (2018) Developmental cognitive neuroscience using latent change score models: a tutorial and applications. Dev Cogn Neurosci 33:99-117.

Klimesch W, Sauseng P, Hanslmayr S (2007) EEG alpha oscillations: the inhibition-timing hypothesis. Brain Res Rev 53:63-88.

Kray J, Lindenberger U (2000) Adult age differences in task switching. Psychol Aging 15:126-147.

LaBar KS, Cook CA, Torpey DC, Welsh-Bohmer KA (2004) Impact of healthy aging on awareness and fear conditioning. Behav Neurosci 118:905-915.

Lee TH, Greening SG, Ueno T, Clewett D, Ponzio A, Sakaki M, Mather M (2018) Arousal increases neural gain via the locus coeruleus-noradrenaline system in younger adults but not in older adults. Nat Hum Behav 2:356-366

Lehrl S (1977) Mehrfachwahl-Wortschatz-Intelligenztest MWT-B. Balingen: Spitta.

Li SC, Lindenberger U, Sikström S (2001) Aging cognition: from neuromodulation to representation. Trends Cogn Sci (Regul Ed) 5:479-486.

Likhtik E, Johansen JP (2019) Neuromodulation in circuits of aversive emotional learning. Nat Neurosci 22:1586-1597.

Lindenberger U, Mayr U (2014) Cognitive aging: is there a dark side to environmental support? Trends Cogn Sci (Regul Ed) 18:7-15.

Liu KY, Acosta-Cabronero J, Cardenas-Blanco A, Loane C, Berry AJ, Betts MJ, Kievit RA, Henson RN, Düzel E, Howard R, Hämmerer D, CamCANc (2019) In vivo visualization of age-related differences in the locus coeruleus. Neurobiol Aging 74:101-111.

Maris E, Oostenveld R (2007) Nonparametric statistical testing of EEG- and MEG-data. J Neurosci Methods 164:177-190.

Marshall JC, Caplan D, Holmes JM (1975) The measure of laterality. Neuropsychologia 13:315-321.

Marzo A, Totah NK, Neves RM, Logothetis NK, Eschenko O (2014) Unilateral electrical stimulation of rat locus coeruleus elicits bilateral response of norepinephrine neurons and sustained activation of medial prefrontal cortex. J Neurophysiol 111:2570-2588.
Mather M, Harley CW (2016) The locus coeruleus: essential for maintaining cognitive function and the aging brain. Trends Cogn Sci (Regul Ed) 20:214-226.

Mather M, Clewett D, Sakaki M, Harley CW (2016) Norepinephrine ignites local hotspots of neuronal excitation: how arousal amplifies selectivity in perception and memory. Behav Brain Sci 39:e200.

McCormick DA (1989) Cholinergic and noradrenergic modulation of thalamocortical processing. Trends Neurosci 12:215-221.

McCormick DA, Pape HC, Williamson A (1991) Actions of norepinephrine in the cerebral cortex and thalamus: implications for function of the central noradrenergic system. Prog Brain Res 88:293-305.

McGinley MJ, David SV, McCormick DA (2015) Cortical membrane potential signature of optimal states for sensory signal detection. Neuron $87: 179-192$

Meredith W, Teresi JA (2006) An essay on measurement and factorial invariance. Med Care 44:S69-S77.

Merz CJ, Kinner VL, Wolf OT (2018) Let's talk about sex: differences in human fear conditioning. Curr Opin Behav Sci 23:7-12.

Mulvey B, Bhatti DL, Gyawali S, Lake AM, Kriaucionis S, Ford CP, Bruchas MR, Heintz N, Dougherty JD (2018) Molecular and functional sex differences of noradrenergic neurons in the mouse locus coeruleus. Cell Rep 23:2225-2235

Neves RM, van Keulen S, Yang M, Logothetis NK, Eschenko O (2018) Locus coeruleus phasic discharge is essential for stimulus-induced gamma oscillations in the prefrontal cortex. J Neurophysiol 119:904920.

Nieuwenhuis S, Aston-Jones G, Cohen JD (2005) Decision making, the P3, and the locus coeruleus-norepinephrine system. Psychol Bull 131:510532 .

Nolan H, Whelan R, Reilly RB (2010) FASTER: fully automated statistical thresholding for EEG artifact rejection. J Neurosci Methods 192:152-162.

Nyberg L, Lövdén M, Riklund K, Lindenberger U, Bäckman L (2012) Memory aging and brain maintenance. Trends Cogn Sci (Regul Ed) 16:292-305.

Oostenveld R, Fries P, Maris E, Schoffelen JM (2011) FieldTrip: open source software for advanced analysis of MEG, EEG, and invasive electrophysiological data. Comput Intell Neurosci 2011:156869.

Passow S, Westerhausen R, Hugdahl K, Wartenburger I, Heekeren HR, Lindenberger U, Li SC (2014) Electrophysiological correlates of adult age differences in attentional control of auditory processing. Cereb Cortex 24:249-260.

Passow S, Westerhausen R, Wartenburger I, Hugdahl K, Heekeren HR, Lindenberger U, Li SC (2012) Human aging compromises attentional control of auditory perception. Psychol Aging 27:99-105.

Perrin F, Pernier J, Bertrand O, Echallier JF (1989) Spherical splines for scalp potential and current density mapping. Electroencephalogr Clin Neurophysiol 72:184-187.

Poli S, Sarlo M, Bortoletto M, Buodo G, Palomba D (2007) Stimulus-preceding negativity and heart rate changes in anticipation of affective pictures. Int J Psychophysiol 65:32-39.

Rasmussen K, Jacobs BL (1986) Single unit activity of locus coeruleus neurons in the freely moving cat: II. Conditioning and pharmacologic studies. Brain Res 371:335-344.

Reimer J, Froudarakis E, Cadwell CR, Yatsenko D, Denfield GH, Tolias AS (2014) Pupil fluctuations track fast switching of cortical states during quiet wakefulness. Neuron 84:355-362.

Reimer J, McGinley MJ, Liu Y, Rodenkirch C, Wang Q, McCormick DA, Tolias AS (2016) Pupil fluctuations track rapid changes in adrenergic and cholinergic activity in cortex. Nat Commun 7:13289.

Robbins TW, Arnsten AF (2009) The neuropsychopharmacology of frontoexecutive function: monoaminergic modulation. Annu Rev Neurosci $32: 267-287$

Safaai H, Neves R, Eschenko O, Logothetis NK, Panzeri S (2015) Modeling the effect of locus coeruleus firing on cortical state dynamics and single-trial sensory processing. Proc Natl Acad Sci USA 112:12834-12839.

Sara SJ, Bouret S (2012) Orienting and reorienting: the locus coeruleus mediates cognition through arousal. Neuron 76:130-141.

Satoh A, Iijima KM (2019) Roles of tau pathology in the locus coeruleus (LC) in age-associated pathophysiology and Alzheimer's disease pathogenesis: potential strategies to protect the LC against aging. Brain Res 1702:1728. 
Schupp HT, Flaisch T, Stockburger J, Junghöfer M (2006) Emotion and attention: event-related brain potential studies. Prog Brain Res 156:31-51.

Schwab S, Helm C (2015) Ûberprüfung von Messinvarianz mittels CFA und DIF-Analysen. Empirische Sonderpädagogik 4845:175-193.

Stitt I, Zhou ZC, Radtke-Schuller S, Fröhlich F (2018) Arousal dependent modulation of thalamo-cortical functional interaction. Nat Commun 9:2455.

Szabadi E (2012) Modulation of physiological reflexes by pain: role of the locus coeruleus. Front Integr Neurosci 6:94.

Szabadi E (2013) Functional neuroanatomy of the central noradrenergic system. J Psychopharmacol 27:659-693.

Thiele A, Bellgrove MA (2018) Neuromodulation of attention. Neuron 97:769-785.

Uematsu A, Tan BZ, Ycu EA, Cuevas JS, Koivumaa J, Junyent F, Kremer EJ, Witten IB, Deisseroth K, Johansen JP (2017) Modular organization of the brainstem noradrenaline system coordinates opposing learning states. Nat Neurosci 20:1602-1611.

van Boxtel GJ, Böcker KB (2004) Cortical measures of anticipation. J Psychophysiol 18:61-76.

van Gerven PW, Paas F, van Merriënboer JJ, Schmidt HG (2004) Memory load and the cognitive pupillary response in aging. Psychophysiology 41:167-174.

Vazey EM, Aston-Jones G (2014) Designer receptor manipulations reveal a role of the locus coeruleus noradrenergic system in isoflurane general anesthesia. Proc Natl Acad Sci USA 111:3859-3864.

Vazey EM, Moorman DE, Aston-Jones G (2018) Phasic locus coeruleus activity regulates cortical encoding of salience information. Proc Natl Acad Sci USA 115:E9439-E9448. von Oertzen T, Brandmaier AM, Tsang S (2015) Structural equation modeling with $\Omega$ nyx. Struct Equ Model 22:148-161.

Voulo ME, Parsons RG (2017) Response-specific sex difference in the retention of fear extinction. Learn Mem 24:245-251.

Wang M, Gamo NJ, Yang Y, Jin LE, Wang XJ, Laubach M, Mazer JA, Lee D, Arnsten AF (2011) Neuronal basis of age-related working memory decline. Nature 476:210-213.

Wechsler D (1981) WAIS-R manual: Wechsler Adult Intelligence ScaleRevised. New York: Psychological Corporation.

Weinshenker D (2018) Long road to ruin: noradrenergic dysfunction in neurodegenerative disease. Trends Neurosci 41:211-223.

Werkle-Bergner M, Shing YL, Müller V, Li SC, Lindenberger U (2009) EEG gamma-band synchronization in visual coding from childhood to old age: evidence from evoked power and inter-trial phase locking. Clin Neurophysiol 120:1291-1302.

Westerhausen R, Moosmann M, Alho K, Medvedev S, Hämäläinen $H$, Hugdahl K (2009) Top-down and bottom-up interaction: manipulating the dichotic listening ear advantage. Brain Res 1250:183-189.

Wilson RS, Nag S, Boyle PA, Hizel LP, Yu L, Buchman AS, Schneider JA, Bennett DA (2013) Neural reserve, neuronal density in the locus ceruleus, and cognitive decline. Neurology 80:1202-1208.

Zerbi V, Floriou-Servou A, Markicevic M, Vermeiren Y, Sturman O, Privitera M, von Ziegler L, Ferrari KD, Weber B, De Deyn PP, Wenderoth N, Bohacek J (2019) Rapid reconfiguration of the functional connectome after chemogenetic locus coeruleus activation. Neuron 103:702-718.e5. 